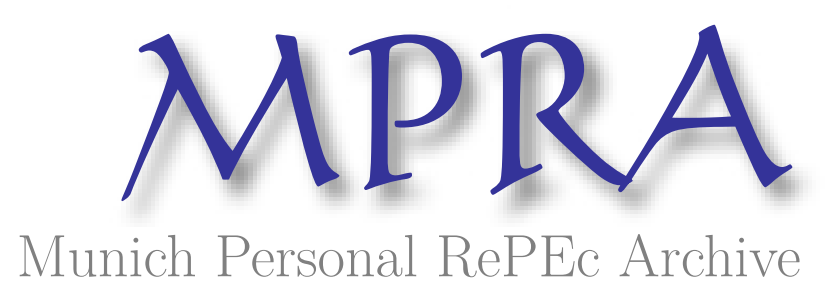

The Barter Method: A New Heuristic for Global Optimization and its Comparison with the Particle Swarm and the Differential Evolution Methods

Mishra, SK

21 October 2006

Online at https://mpra.ub.uni-muenchen.de/543/

MPRA Paper No. 543, posted 21 Oct 2006 UTC 


\title{
The Barter Method: A New Heuristic for Global Optimization and its Comparison with the Particle Swarm and the Differential Evolution Methods
}

\author{
SK Mishra \\ Department of Economics \\ North-Eastern Hill University \\ Shillong, Meghalaya (India)
}

Introduction: The objective of this paper is to introduce a new population-based (stochastic) heuristic to search the global optimum of a (continuous) multi-modal function and to assess its performance (on a fairly large number of benchmark functions) vis-à-vis that of two other well-established and very powerful methods, namely, the Particle Swarm (PS) and the Differential Evolution (DE) methods of global optimization. We will call this new method the Barter Method of global optimization.

For the purpose of brevity we would not present here any introductory note on the Particle Swarm (or the Modified Repulsive Particle Swarm, MRPS, variant that we have used in this study) or the DE method. Such a note is available elsewhere [Mishra, 2006 (d) and (f)]. Additionally, there is a large literature on these methods.

The Barter Method: This method is based on the well-known proposition in welfare economics that competitive equilibria, under fairly general conditions, tend to be Pareto optimal [Takayama, 1974, pp. 185-201]. In its simplest version, implementation of this proposition may be outlined as follows:

Let there be $n$ (fairly large number of) individuals in a population and let each individual, $i$, own (or draw from the environment) an $m$-element real vector of resources, $x_{i}=\left(x_{i 1}, x_{i 2}, \ldots, x_{i m}\right)$. For every $x_{i}$ there is a (single-valued) function $f\left(x_{i}\right)$ that may be used as a measure of the worth of $x_{i}$ that the individual would like to optimize. The optimand function $f($.$) is unique and common to all the individuals. Now, let the$ individuals in the (given) population enter into a barter of their resources with the condition that (i) $\beta\left(x_{i j}, x_{k \ell}: i \neq k ; j \neq l\right)$ or a transaction is feasible across different persons and different resources only, and (ii) the resources will change hands (materialize) only if such a transaction is beneficial to (more desired by) both the parties (in the barter). The choice of the individuals, $(i, k)$ and the resources, $(j, \ell)$ in every transaction and the quantum of transaction would be stochastic in nature. If such transactions are allowed for a large number of times, then at the end of the session: (a) every individual would be better off than what he was at the initial position, and (b) at least one individual would reach the global optimum.

A Computer Program: A computer program (FORTRAN) that works out the global optimum of the test functions by the three methods (MRPS, DE and Barter) is appended. It incorporated 75 benchmark functions of varied types, some well-known and others new (proposed by the present author). 
The Findings: In all, benchmark functions have been optimized 77 times. As presented in table-1, the DE succeeds in 70 cases, the RPS succeeds in 60 cases, while the Barter method succeeds for a modest number of 51 cases. The DE as well as Barter methods are unstable for stochastic functions (Yao-Liu\#7 and Fletcher-Powell functions). In eight cases, the Barter method could not converge in 10000 iterations (due to slow convergence rate), while in 4 cases the MRPS could not converge.

\begin{tabular}{|c|c|c|c|c|c|c|c|c|c|}
\hline \multirow[t]{2}{*}{ Test Function } & \multirow{2}{*}{\begin{tabular}{|l|} 
Dim \\
(M)
\end{tabular}} & \multicolumn{3}{|c|}{ Success } & \multirow[t]{2}{*}{ Test Function } & \multirow{2}{*}{$\begin{array}{l}\text { Dim } \\
\text { (M) }\end{array}$} & \multicolumn{3}{|c|}{ Success } \\
\hline & & DE & BA & PS & & & BA & DE & PS \\
\hline New Fn \#1 & 2 & \begin{tabular}{|l|} 
yes \\
\end{tabular} & no & nc & Fenton-Eason Fn & 2 & yes & yes & yes \\
\hline New Fn \#2 & 2 & yes & no & yes & Hougen Fn & 5 & yes & no & no \\
\hline New Fn \#3 & 2 & yes & yes & yes & Giunta Fn & 2 & yes & yes & yes \\
\hline New Fn \#4 & 2 & yes & yes & yes & Egg-Holder Fn & 2 & yes & yes & yes \\
\hline New Fn \#8 & 2 & yes & yes & yes & Trid Fn & 10 & yes & $\mathrm{nc}$ & yes \\
\hline Quintic Fn & 10 & yes & no & no & Greiwank Fn & 10 & yes & $\mathrm{nc}$ & $\mathrm{nc}$ \\
\hline Needle-eye Fn & 10 & yes & no & no & Weierstrass Fn & 10 & yes & yes & no \\
\hline Fletcher-Powell Fn (o) & 5 & yes & no & yes & Levy\#3 Fn & 2 & yes & yes & yes \\
\hline Fletcher-Powell Fn (1) & 5 & uns & uns & yes & Levy\#5 Fn & 2 & yes & yes & yes \\
\hline Fletcher-Powell Fn (2) & 5 & uns & uns & yes & Levy\#8 Fn & 3 & yes & yes & yes \\
\hline Powell Fn & 8 & yes & yes & yes & Colville Fn. & 4 & yes & yes & yes \\
\hline Glankwahmdee Fn & 5 & yes & yes & yes & Hartmann Fn & 3 & yes & yes & yes \\
\hline Zero-sum Fn & 10 & yes & no & no & Rastrigin Fn & 10 & yes & yes & no \\
\hline Corana Fn & 4 & yes & yes & yes & Ackley Fn & 10 & yes & yes & yes \\
\hline Mod RCos Fn & 2 & yes & yes & yes & Michalewicz Fn & 10 & yes & $\mathrm{nc}$ & yes \\
\hline Freud-Roth Fn & 2 & yes & yes & yes & Schwefel Fn & 10 & yes & $\mathrm{nc}$ & yes \\
\hline Anns XOR Fn & 9 & yes & no & no & Shubert Fn & 2 & yes & yes & yes \\
\hline Perm \#1 Fn & 4 & yes & $\mathrm{nc}$ & yes & Dixon-Price Fn & 10 & no & no & no \\
\hline Perm \#2 Fn & 5 & no & no & no & Shekel Fn & 4 & yes & no & yes \\
\hline Power-Sum Fn & 4 & no & yes & yes & Paviani Fn & 10 & yes & yes & yes \\
\hline Goldstein-Price Fn & 2 & yes & yes & yes & Branin\#1 Fn & 2 & yes & yes & yes \\
\hline Bukin-6 Fn & 2 & no & no & no & Branin\#2 Fn & 2 & yes & yes & yes \\
\hline DCS Fn & 4 & yes & no & yes & Bohachevsky\#1 Fn & 2 & yes & yes & yes \\
\hline New Factorial Fn & 4 & yes & no & yes & Bohachevsky\#2 Fn & 2 & yes & yes & yes \\
\hline New Decanomial Fn & 2 & \begin{tabular}{|l|} 
yes \\
\end{tabular} & yes & yes & Bohachevsky\#3 Fn & 2 & yes & yes & yes \\
\hline Judge Fn & 2 & yes & yes & yes & Easom Fn & 2 & yes & yes & yes \\
\hline New Dodecal Fn & 3 & yes & yes & yes & Rosenbrock Fn & 10 & yes & nc & yes \\
\hline New sum=prod Fn & 2 & yes & yes & no & Crosslegged Table Fn & 2 & yes & yes & no \\
\hline New AM=GM Fn & 10 & yes & no & yes & Cross Fn & 2 & yes & yes & yes \\
\hline Yao-Liu\#2 Fn & 10 & yes & yes & nc & Cross-in-Tray Fn & 2 & yes & yes & yes \\
\hline Yao-Liu\#3 Fn & 10 & yes & yes & yes & Crowned Cross Fn & 2 & yes & yes & no \\
\hline Yao-Liu\#4 Fn & 10 & yes & $\mathrm{nc}$ & $\mathrm{nc}$ & TT-Holder Fn & 2 & yes & yes & yes \\
\hline Yao-Liu\#6 Fn & 10 & yes & yes & yes & Holder Table Fn & 2 & yes & yes & yes \\
\hline Yao-Liu\#7 Fn & 10 & uns & uns & yes & Carrom Table Fn & 2 & yes & yes & yes \\
\hline Yao-Liu\#12 Fn & 10 & yes & yes & yes & Pen-Holder Fn & 2 & yes & yes & yes \\
\hline Yao-Liu\#13 Fn & 10 & yes & yes & yes & Bird Fn & 2 & yes & yes & yes \\
\hline Yao-Liu\#14 Fn & 2 & yes & yes & yes & Chichinadze Fn & 2 & yes & yes & yes \\
\hline Yao-Liu\#15 Fn & 4 & yes & $\mathrm{nc}$ & yes & McCormick Fn & 2 & yes & yes & yes \\
\hline Wood's Fn & 4 & yes & yes & yes & No. of Failure in 77 trials & & 7 & 26 & 17 \\
\hline
\end{tabular}


Seen as such, the barter method is inferior to the other two methods. Additionally, the convergence rate of the Barter method is slower than the DE as well as the MRPS. However, the DE and the RPS have a history of a full decade behind them and they have been improved many times. In the present exercise, the RPS is a modified version (MRPS) that has an extra ability for local search. The DE version used here uses the latest (available) schemes of crossover, mutation and recombination. In comparison to this, the Barter method is a nascent one. We need a thorough investigation into the nature and performance of the Barter method.

\section{Bibliography}

- Bauer, J.M.: "Harnessing the Swarm: Communication Policy in an Era of Ubiquitous Networks and Disruptive Technologies", Communications and Strategies, 45, 2002.

- Box, M.J.: "A new method of constrained optimization and a comparison with other methods". Comp. J. 8, pp. 42-52, 1965.

- Bukin, A. D.: New Minimization Strategy For Non-Smooth Functions, Budker Institute of Nuclear Physics preprint BUDKER-INP-1997-79, Novosibirsk 1997.

- Cerny, V.: "Thermodynamical Approach to the Traveling Salesman Problem: An Efficient Simulation Algorithm", J. Opt. Theory Appl., 45, 1, 41-51, 1985.

- Eberhart R.C. and Kennedy J.: "A New Optimizer using Particle Swarm Theory", Proceedings Sixth Symposium on Micro Machine and Human Science, pp. 39-43. IEEE Service Center, Piscataway, NJ, 1995.

- Fleischer, M.: "Foundations of Swarm Intelligence: From Principles to Practice", Swarming Network Enabled C4ISR, arXiv:nlin.AO/0502003 v1 2 Feb 2005.

- G.E.P. Box, "Evolutionary operation: A method for increasing industrial productivity", Applied Statistics, 6, pp. 81-101, 1957.

- Glover F.," Future paths for Integer Programming and Links to Artificial Intelligence", Computers and Operations Research, 5:533-549, 1986.

- Hayek, F.A.: The Road to Serfdom, Univ. of Chicago Press, Chicago, 1944.

- Holland, J.: Adaptation in Natural and Artificial Systems, Univ. of Michigan Press, Ann Arbor, 1975.

- Karush, W. Minima of Functions of Several Variables with Inequalities as Side Constraints. M.Sc. Dissertation. Dept. of Mathematics, Univ. of Chicago, Chicago, Illinois, 1939.

- Kirkpatrick, S., Gelatt, C.D. Jr., and Vecchi, M.P.: "Optimization by Simulated Annealing", Science, 220, 4598, 671-680, 1983.

- Kuhn, H.W. and Tucker, A.W.: "Nonlinear Programming", in Neymann, J. (ed) Proceedings of Second Berkeley Symposium on Mathematical Statistics and Probability, Univ. of California Press, Berkrley, Calif. pp. 481-492, 1951.

- Metropolis, N. The Beginning of the Monte Carlo Method. Los Alamos Science, No. 15, Special Issue, pp. 125-130, 1987.

- Metropolis, N., Rosenbluth, A., Rosenbluth, M., Teller, A., and Teller, E.: "Equation of State Calculations by Fast Computing Machines", J. Chem. Phys.,21, 6, 1087-1092, 1953.

- Mishra, S.K.: "Some Experiments on Fitting of Gielis Curves by Simulated Annealing and Particle Swarm Methods of Global Optimization”, Social Science Research Network (SSRN): http://ssrn.com/abstract=913667, Working Papers Series, 2006 (a). 
- Mishra, S.K.: "Least Squares Fitting of Chacón-Gielis Curves by the Particle Swarm Method of Optimization", Social Science Research Network (SSRN), Working Papers Series, http://ssrn.com/abstract=917762, 2006 (b).

- Mishra, S.K.: "Performance of Repulsive Particle Swarm Method in Global Optimization of Some Important Test Functions: A Fortran Program" , Social Science Research Network (SSRN), Working Papers Series, http://ssrn.com/abstract=924339, 2006 (c).

- Mishra, S.K.: "Some New Test Functions for Global Optimization and Performance of Repulsive Particle Swarm Method", Social Science Research Network (SSRN) Working Papers Series, http://ssrn.com/abstract=927134, 2006 (d).

- Mishra, S.K.: "Repulsive Particle Swarm Method on Some Difficult Test Problems of Global Optimization" ,SSRN: http://ssrn.com/abstract=928538, 2006 (e).

- Mishra, SK.: "Global Optimization by Differential Evolution and Particle Swarm Methods: Evaluation on Some Benchmark Functions" SSRN: http://ssrn.com/abstract=933827,2006 (f)

- Nagendra, S.: Catalogue of Test Problems for Optimization Algorithm Verification, Technical Report 97-CRD-110, General Electric Company, 1997.

- Nelder, J.A. and Mead, R.: "A Simplex method for function minimization" Computer Journal, 7: pp. 308-313, 1964.

- Parsopoulos, K.E. and Vrahatis, M.N., "Recent Approaches to Global Optimization Problems Through Particle Swarm Optimization", Natural Computing, 1 (2-3), pp. 235- 306, 2002.

- Prigogine, I. and Strengers, I.: Order Out of Chaos: Man's New Dialogue with Nature, Bantam Books, Inc. NY, 1984.

- Silagadge, Z.K.: "Finding Two-Dimensional Peaks", Working Paper, Budkar Insttute of Nuclear Physics, Novosibirsk, Russia, arXive:physics/0402085 V3 11 Mar 2004.

- Simon, H.A.: Models of Bounded Rationality, Cambridge Univ. Press, Cambridge, MA, 1982.

- Smith, A.: The Theory of the Moral Sentiments, The Adam Smith Institute (2001 e-version), 1759.

- Storn, R. and Price, K: "Differential Evolution - A simple and Efficient Adaptive Scheme for Global Optimization over Continuous Spaces" : Technical Report, International Computer Science Institute, Berkley, 1995.

- Sumper, D.J.T.: "The Principles of Collective Animal Behaviour", Phil. Trans. R. Soc. B. 361, pp. 5-22, 2006.

- Takayama, A.: Mathematical Economics, The Dryden Press, Hinsdale, Illinois, 1974.

- Törn, A.A and Viitanen, S.: "Topographical Global Optimization using Presampled Points", J. of Global Optimization, 5, pp. 267-276, 1994.

- Törn, A.A.: "A search Clustering Approach to Global Optimization", in Dixon, LCW and Szegö, G.P. (Eds) Towards Global Optimization - 2, North Holland, Amsterdam, 1978.

- Tsallis, C. and Stariolo, D.A.: "Generalized Simulated Annealing", ArXive condmat/9501047 v1 12 Jan, 1995.

- Valentine, R.H.: Travel Time Curves in Oblique Structures, Ph.D. Dissertation, MIT, Mass, 1937.

- Veblen, T.B.: "Why is Economics Not an Evolutionary Science" The Quarterly Journal of Economics, 12, 1898.

- Veblen, T.B.: The Theory of the Leisure Class, The New American library, NY. (Reprint, 1953), 1899.

- Vesterstrøm, J. and Thomsen, R.: “A comparative Study of Differential Evolution, Particle Swarm Optimization, and Evolutionary Algorithms on Numerical Benchmark Problems", Congress on Evolutionary Computation, 2004. CEC2004, 2, pp. 1980-1987, 2004.

- Whitley, D., Mathias, K., Rana, S. and Dzubera, J.: "Evaluating Evolutionary Algorithms", Artificial Intelligence, 85, pp. 245-276, 1996.

- Yao, X. and Liu, Y.: "Fast Evolutionary Programming", in Fogel, LJ, Angeline, PJ and Bäck, T (eds) Proc. $5^{\text {th }}$ Annual Conf. on Evolutionary programming, pp. 451-460, MIT Press, Mass, 1996. 


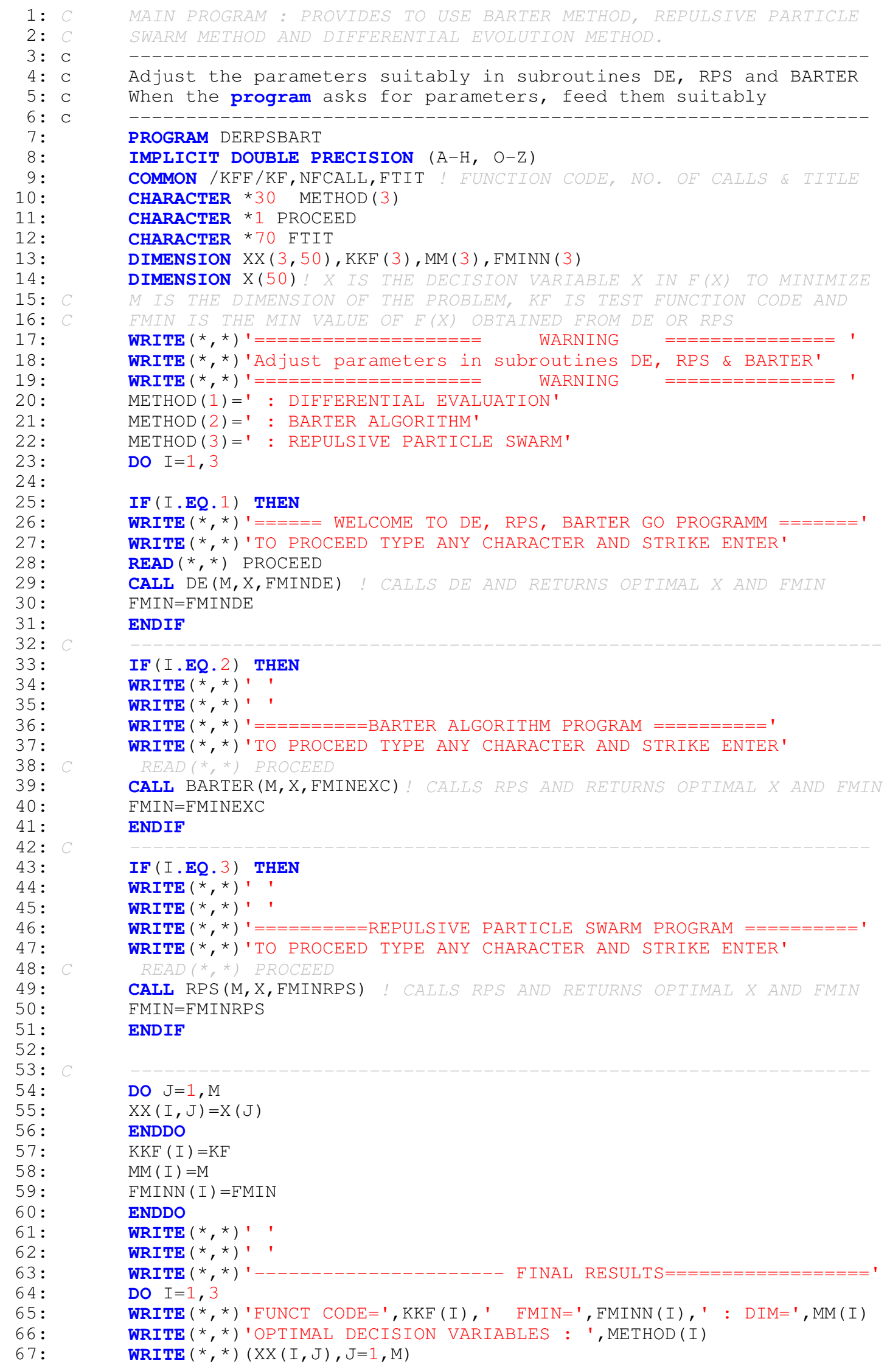




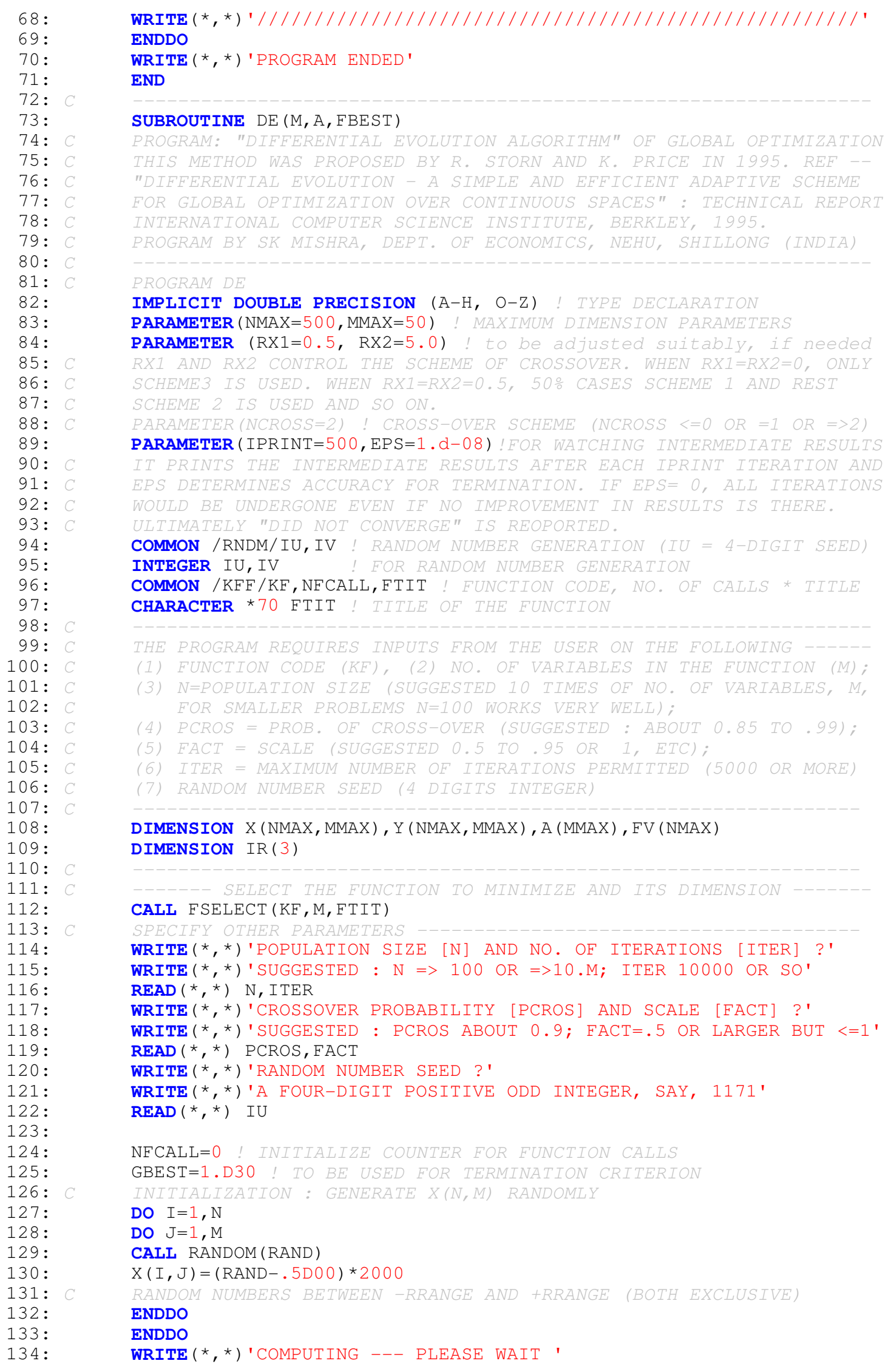




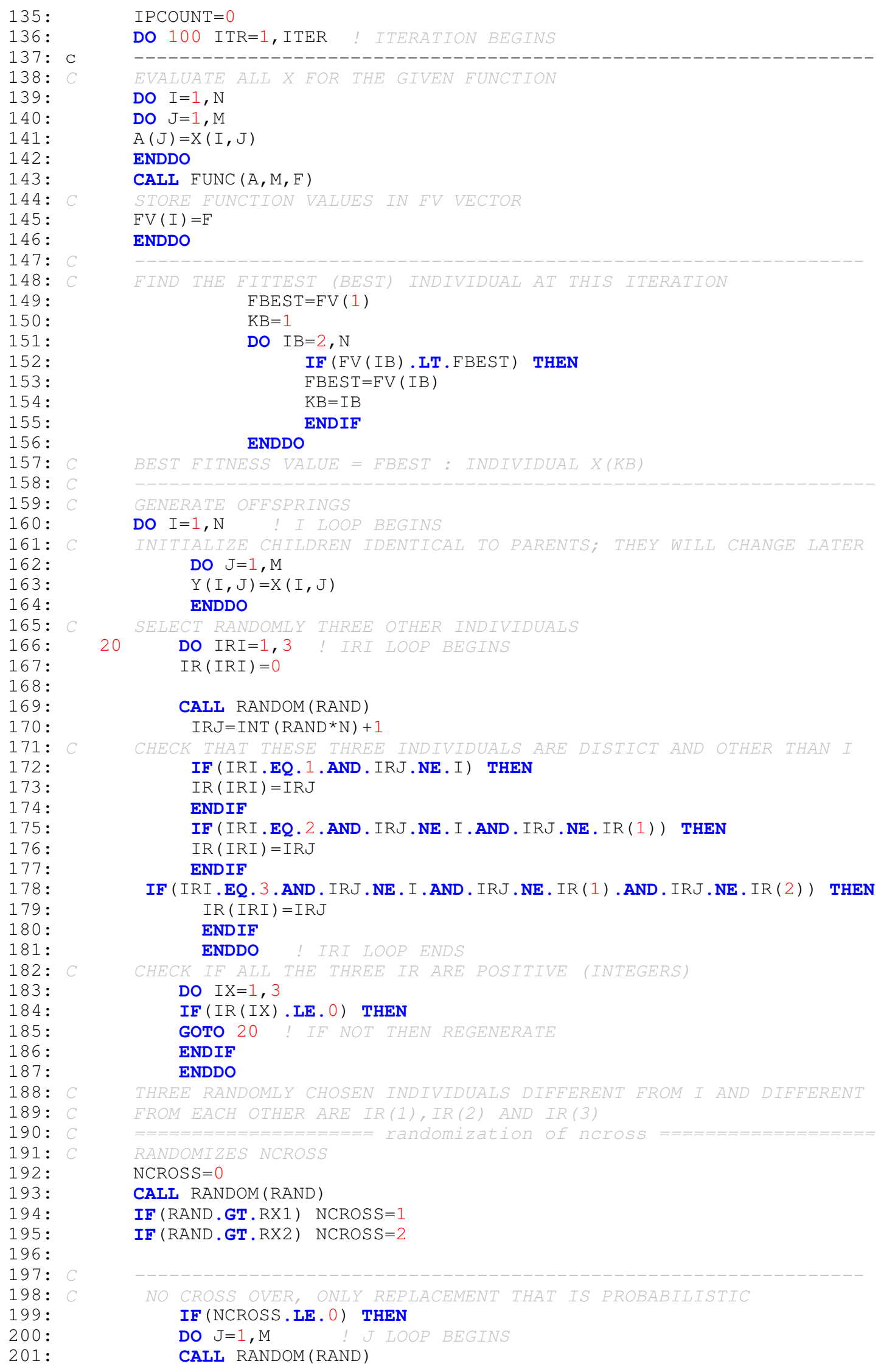


202:

203:

$204:$

205 :

206

207:

208:

209:

210:

211:

212:

213:

214:

215:

216:

217:

218:

219:

220:

221:

222

223:

$224:$

225:

226 :

227:

228:

229:

230:

231:

232:

233:

234

235

236:

$237:$

238:

239:

240 :

241:

242:

243:

244 :

$245:$

246 :

$247:$

248:

249:

250 :

251:

252:

253:

254:

255:

256:

257:

258:

259:

260 :

261:

262:

263:

264:

265:

266 :

267:

268:

IF (RAND. LE.PCROS) THEN ! REPLACE IF RAND $<P C R O S$

$\mathrm{A}(\mathrm{J})=\mathrm{X}(\operatorname{IR}(1), \mathrm{J})+(\mathrm{X}(\operatorname{IR}(2), \mathrm{J})-\mathrm{X}(\operatorname{IR}(3), \mathrm{J})) * \mathrm{FACT} !$ CANDIDATE CHILD

ENDIF

ENDDO ! J LOOP ENDS

ENDIF

CROSSOVER SCHEME (EXPONENTIAL) SUGGESTED BY KENNETH PRICE IN HIS

PERSONAL LETTER TO THE AUTHOR (DATED SEPTEMBER 29, 2006 )

IF (NCROSS .EQ.1) THEN

CALL RANDOM(RAND)

$1 \quad \mathrm{JR}=\mathrm{INT}(\mathrm{RAND} * \mathrm{M})+1$

$\mathrm{J}=\mathrm{JR}$

$2 A(J)=X(I R(1), J)+F A C T *(X(I R(2), J)-X(I R(3), J))$

$3 \quad \mathrm{~J}=\mathrm{J}+1$

IF ( J.GT.M) J=1

4 IF (J.EQ.JR) GOTO 10

5 CALL RANDOM (RAND)

IF (PCROS. LE. RAND) GOTO 2

$6 \quad \mathrm{~A}(\mathrm{~J})=\mathrm{X}(\mathrm{I}, \mathrm{J})$

$7 \quad \mathrm{~J}=\mathrm{J}+1$

IF ( J . GT .M) $J=1$

IF (J.EQ.JR) GOTO 10

GOTO 6

10 CONTINUE

ENDIF

CROSSOVER SCHEME (NEW) SUGGESTED BY KENNETH PRICE IN HIS

PERSONAL IETTER TO THE AUTHOR (DATED OCTOBER 18, 2006)

IF (NCROSS.GE. 2) THEN

CALL RANDOM(RAND)

IF (RAND . LE. PCROS) THEN

CALL NORMAL (RN)

DO $J=1, M$

$A(J)=X(I, J)+(X(I R(I), J)+X(I R(2), J)-2 * X(I, J)) * R N$

ENDDO

ELSE

DO $J=1, M$

$A(J)=X(I, J)+(X(I R(1), J)-X(I R(2), J)) !$ FACT ASSUMED TO BE ENDDO

ENDIF

ENDIF

CALL FUNC (A, M, F) ! EVALUATE THE OFFSPRING

IF (F.LT.FV(I)) THEN! IF BETTER, REPLACE PARENTS BY THE CHILD

$\mathrm{FV}(\mathrm{I})=\mathrm{F}$

DO $\mathrm{J}=1, \mathrm{M}$

$\mathrm{Y}(\mathrm{I}, \mathrm{J})=\mathrm{A}(\mathrm{J})$

ENDDO

ENDIF

ENDDO

DO $I=1, N$

DO $J=1, M$

$\mathrm{X}(\mathrm{I}, \mathrm{J})=\mathrm{Y}(\mathrm{I}, \mathrm{J})$ ! NEW GENERATION IS A MIX OF BETTER PARENTS AND

ENDDO

ENDDO

IPCOUNT $=$ IPCOUNT +1

IF ( IPCOUNT .EQ.IPRINT) THEN

DO $\mathrm{J}=1, \mathrm{M}$

$A(J)=X(K B, J)$

ENDDO

$\operatorname{WRITE}(*, *)(\mathrm{X}(\mathrm{KB}, \mathrm{J}), \mathrm{J}=1, \mathrm{M}), '$ FBEST UPTO NOW $=$ ', FBEST

WRITE $(*, *)$ 'TOTAL NUMBER OF FUNCTION CALLS $=$ ', NFCALL

IF (DABS (FBEST-GBEST) . LT.EPS) THEN

$\operatorname{WRITE}(*, *) \operatorname{FTIT}$

WRITE $(*, *)$ 'COMPUTATION OVER' 
269:

270 :

271:

272:

273:

274 :

275:

276:

277:

278:

279:

280:

281:

282:

283:

284 :

285:

286:

287:

288:

289:

290:

291:

292:

293:

294 :

295:

296:

297:

298:

299:

300 :

$301:$

302:

303:

$304:$

305:

306 :

307 :

308:

309 :

310:

311:

312:

313:

314:

315:

316:

317:

318:

319:

320 :

321 :

322:

323:

324 :

325 :

326 :

$327:$

328 :

329:

330 :

331:

332:

333:

$334:$

335:

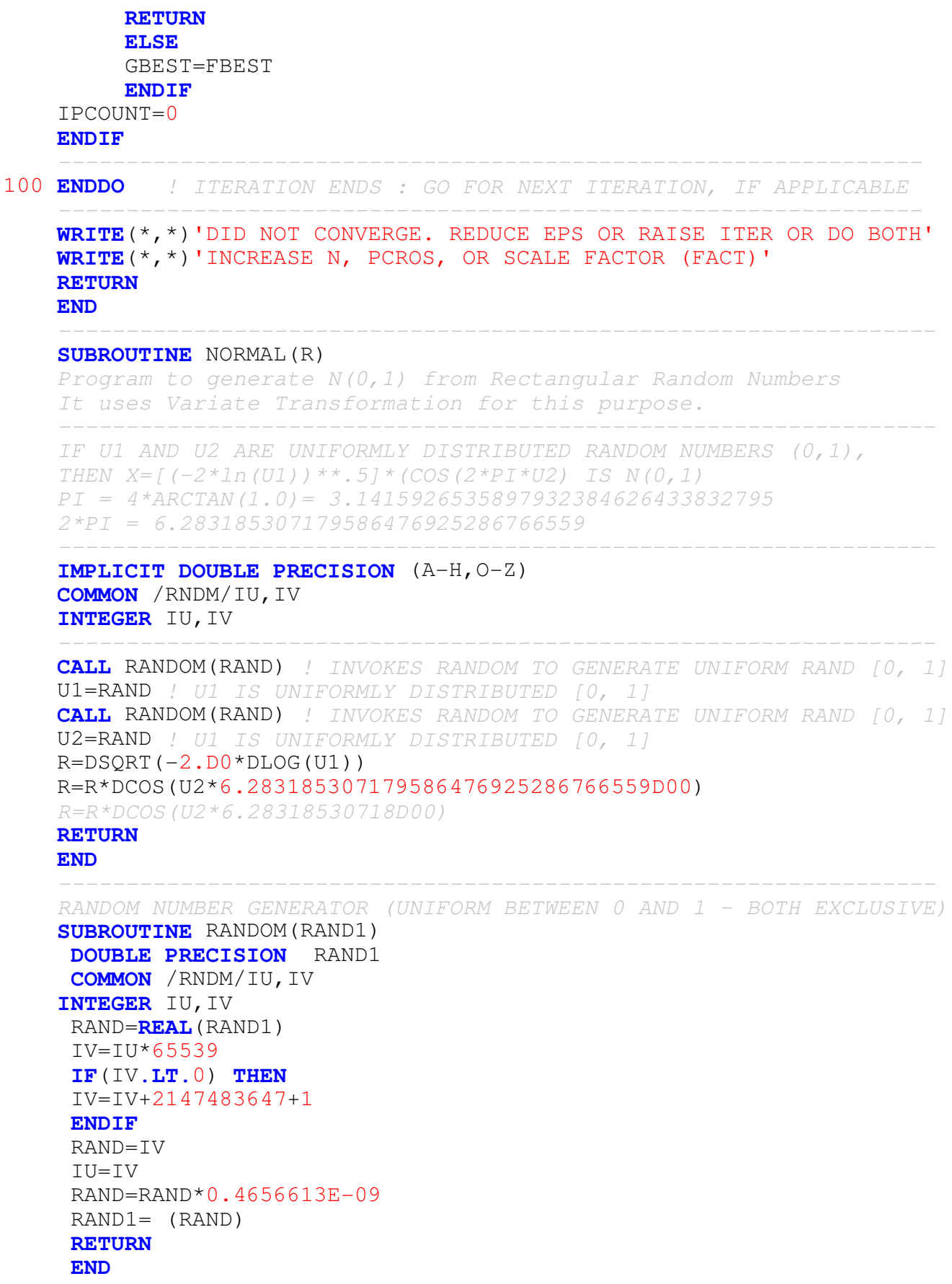


336 :

$337:$

338:

339 :

340 :

341 :

342 :

343:

344 :

$345:$

346 :

347 :

$348:$

349 :

350 :

351 :

352:

353:

354 :

$355:$

$356:$

357:

358:

359:

360 :

361 :

362:

363:

364 :

$365:$

366 :

$367:$

$368:$

$369:$

370 :

371:

372:

373 :

374:

375 :

376 :

377:

378:

$379:$

380 :

$381:$

382:

383:

$384:$

385:

386 :

387 :

$388:$

389:

390 :

391 :

392:

393:

394 :

395:

396 :

397:

398:

$399:$

400 :

401:

402:

MAY BE INCREASED TO 50 OR 100 TOO. THE PARAMETER NN IS THE SIZE OF RANDOMLY CHOSEN NEIGHBOURS. 15 TO 25 (BUT SUFFICIENTLY LESS THAN N) IS A GOOD CHOICE. MX IS THE MAXIMAL SIZE OF DECISION VARIABLES. IN $F(X I, X 2, \ldots, X M) M$ SHOULD BE LESS THAN OR EQUAL TO MX. ITRN IS THE NO. OF ITERATIONS. IT MAY DEPEND ON THE PROBLEM. 200 (AT LEAST) TO 500 ITERATIONS MAY BE GOOD ENOUGH. BUT FOR FUNCTIONS LIKE ROSENBROCKOR GRIEWANK OF LARGE SIZE (SAY M=3O) IT IS NEEDED THAT ITRN IS LARGE, SAY 5000 OR EVEN 10000. SIGMA INTRODUCES PERTURBATION \& HELPS THE SEARCH JUMP OUT OF LOCAI OPTIMA. FOR EXAMPLE : RASTRIGIN FUNCTION OF DMENSION 30 OR LARGER NSTEP DOES LOCAL SEARCH BY TUNNELLING AND WORKS WELL BETWEEN 5 AND 15, WHICH IS MUCH ON THE HIGHER SIDE. ITOP $<=1$ (RING); ITOP=2 (RING AND RANDOM); ITOP=>3 (RANDOM) NSIGMA $=0$ (NO CHAOTIC PERTURBATION); NSIGMA=I (CHAOTIC PERTURBATION) NOTE THAT NSIGMA=I NEED NOT ALWAYS WORK BETTER (OR WORSE) 


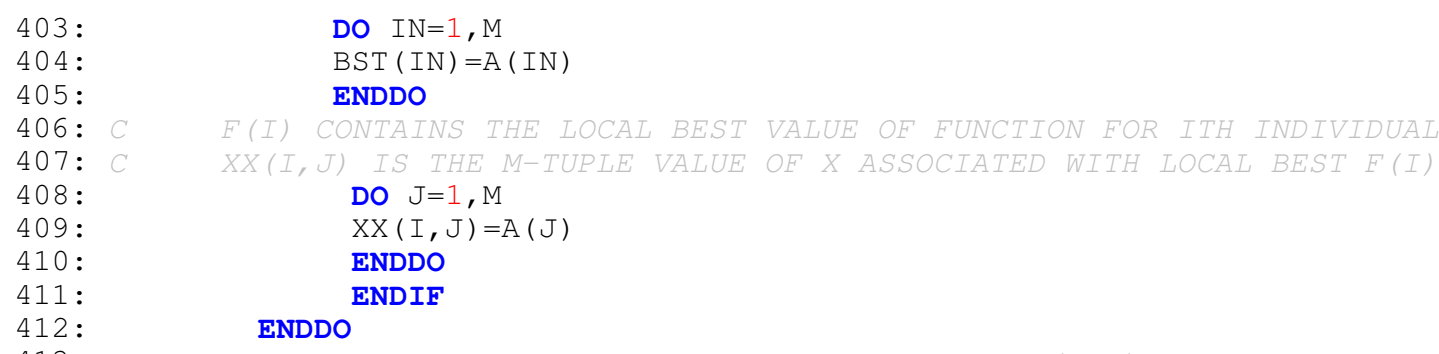




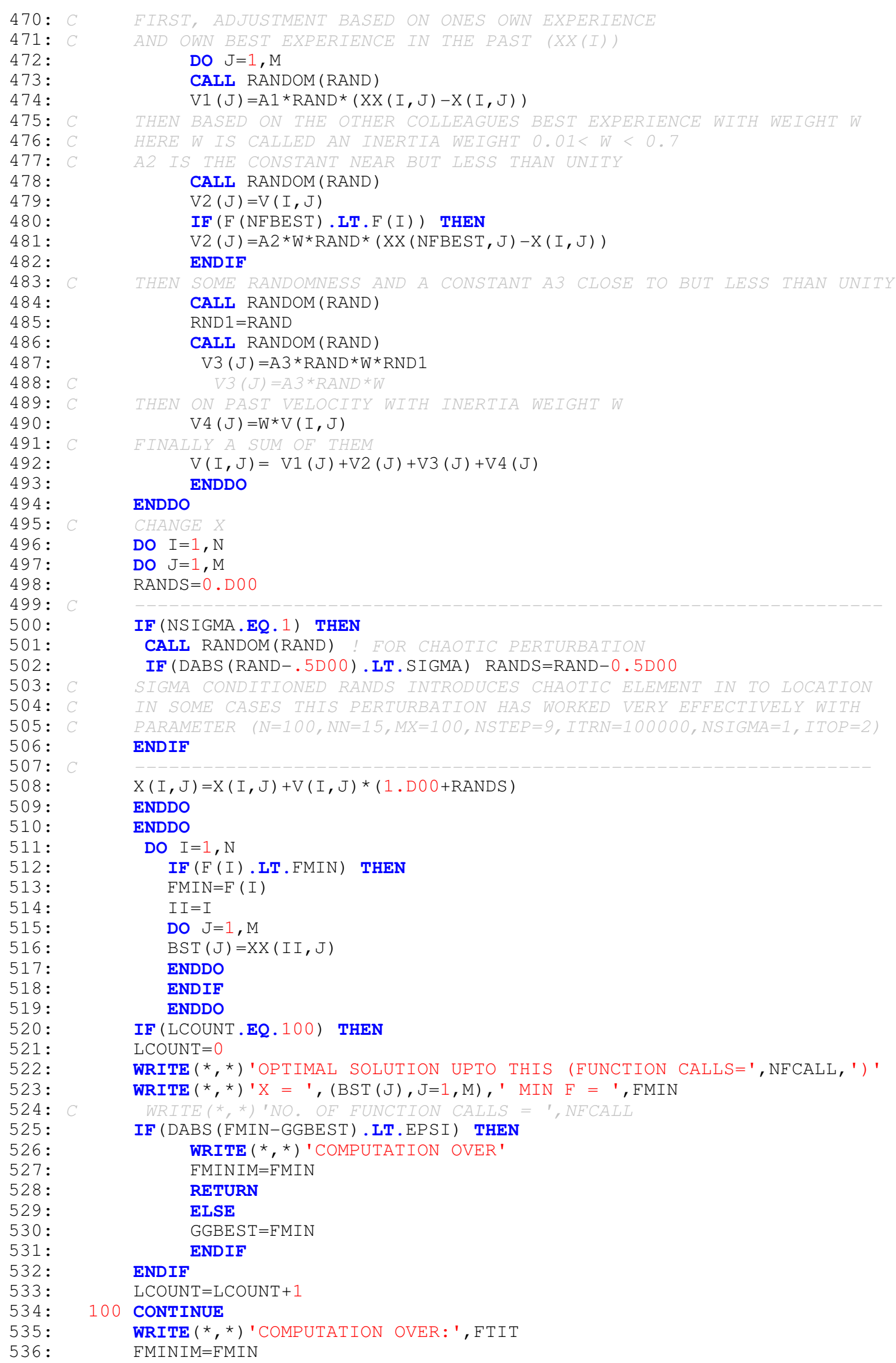

$470:$

471:

472:

473:

474:

475:

476 :

477:

478:

479:

480 :

$481:$

482:

483:

484 :

485:

486 :

487:

488:

489:

490 :

491:

492:

493:

$494:$

495:

496:

497:

498:

499:

500:

501:

502:

503 :

504 :

505:

506:

507:

508:

509:

510:

511:

512:

513:

514:

515:

516:

517:

518:

519:

520:

521:

522:

523 :

524:

525:

526:

527:

528:

529:

530 :

531:

532:

533:

534 :

535:

536:

FIRST, ADJUSTMENT BASED ON ONES OWN EXPERIENCE

AND OWN BEST EXPERIENCE IN THE PAST (XX(I))

DO $\mathrm{J}=1, \mathrm{M}$

CALL RANDOM(RAND)

$\mathrm{V} 1(\mathrm{~J})=\mathrm{A} 1$ *RAND* $(\mathrm{XX}(\mathrm{I}, \mathrm{J})-\mathrm{X}(\mathrm{I}, \mathrm{J}))$

THEN BASED ON THE OTHER COLLEAGUES BEST EXPERIENCE WITH WEIGHT W

HERE W IS CALLED AN INERTIA WEIGHT $0.01<W<0.7$

A2 IS THE CONSTANI NEAR BUT LESS THAN UNITY

CALL RANDOM (RAND)

$\mathrm{V} 2(\mathrm{~J})=\mathrm{V}(\mathrm{I}, \mathrm{J})$

IF (F (NFBEST) .LT.F (I)) THEN

$\mathrm{V} 2(\mathrm{~J})=\mathrm{A} 2{ }^{*} \mathrm{~W}^{\star}$ RAND $*(\mathrm{XX}(\operatorname{NFBEST}, \mathrm{J})-\mathrm{X}(\mathrm{I}, \mathrm{J}))$

ENDIF

THEN SOMF RANDOMNESS AND A CONSTANT A3 CLOSE TO BUT LESS THAN UNITY

CALL RANDOM(RAND)

RND1 $=$ RAND

CALL RANDOM(RAND)

$\mathrm{V} 3(\mathrm{~J})=\mathrm{A} 3 * \mathrm{RAND} * \mathrm{~W} * \mathrm{RND} 1$

$V 3(J)=A 3 * R A N D * W$

THEN ON PAST VELOCITY WITH INERTIA WEIGHT W

$\mathrm{V} 4(\mathrm{~J})=\mathrm{W} * \mathrm{~V}(\mathrm{I}, \mathrm{J})$

FINALIY A SUM OF THEM

$\mathrm{V}(\mathrm{I}, \mathrm{J})=\mathrm{V} 1(\mathrm{~J})+\mathrm{V} 2(\mathrm{~J})+\mathrm{V} 3(\mathrm{~J})+\mathrm{V} 4(\mathrm{~J})$

ENDDO

ENDDO

DO $I=1, N$

DO $\mathrm{J}=1, \mathrm{M}$

RANDS $=0$. D 00

IF (NSIGMA.EQ.1) THEN

CALL RANDOM (RAND)

IF (DABS (RAND- . 5D00) . LT.SIGMA) RANDS=RAND-0.5DO0

SIGMA CONDITIONED RANDS INTRODUCES CHAOTIC ELEMENT IN TO LOCATION IN SOME CASES THIS PERTURBATION HAS WORKED VERY EFFECTIVELY WITH PARAMETER $(N=100, N N=15, M X=100, N S T E P=9, I T R N=100000, N S I G M A=1, I T O P=2)$ ENDIF

$X(I, J)=X(I, J)+V(I, J) *(1 . D 00+R A N D S)$

ENDDO

ENDDO

DO $I=1, N$

IF (F (I) . LT.FMIN) THEN

$F M I N=F(I)$

$I I=I$

DO $\mathrm{J}=1, \mathrm{M}$

$\operatorname{BST}(J)=X X(I I, J)$

ENDDO

ENDIF

ENDDO

IF ( LCOUNT .EQ.100) THEN

$\mathrm{LCOUNT}=0$

WRITE $(*, *)$ 'OPTIMAL SOLUTION UPTO THIS (FUNCTION CALLS=', NFCALL, ') '

$\operatorname{WRITE}(*, *) ' \mathrm{X}={ }^{\prime},(\operatorname{BST}(\mathrm{J}), \mathrm{J}=1, \mathrm{M}),{ }^{\prime} \operatorname{MIN} \mathrm{F}=$ ', $\operatorname{FMIN}$

IF (DABS (FMIN-GGBEST) .LT.EPSI) THEN

WRITE $(*, *)$ 'COMPUTATION OVER'

FMINIM=FMIN

RETURN

ELSE

GGBEST $=\mathrm{FMIN}$

ENDIF

ENDIF

$\mathrm{LCOUNT}=\mathrm{LCOUNT}+1$

100 CONTINUE

WRITE $(*, *)$ 'COMPUTATION OVER: ',FTIT

FMINIM=EMIN 


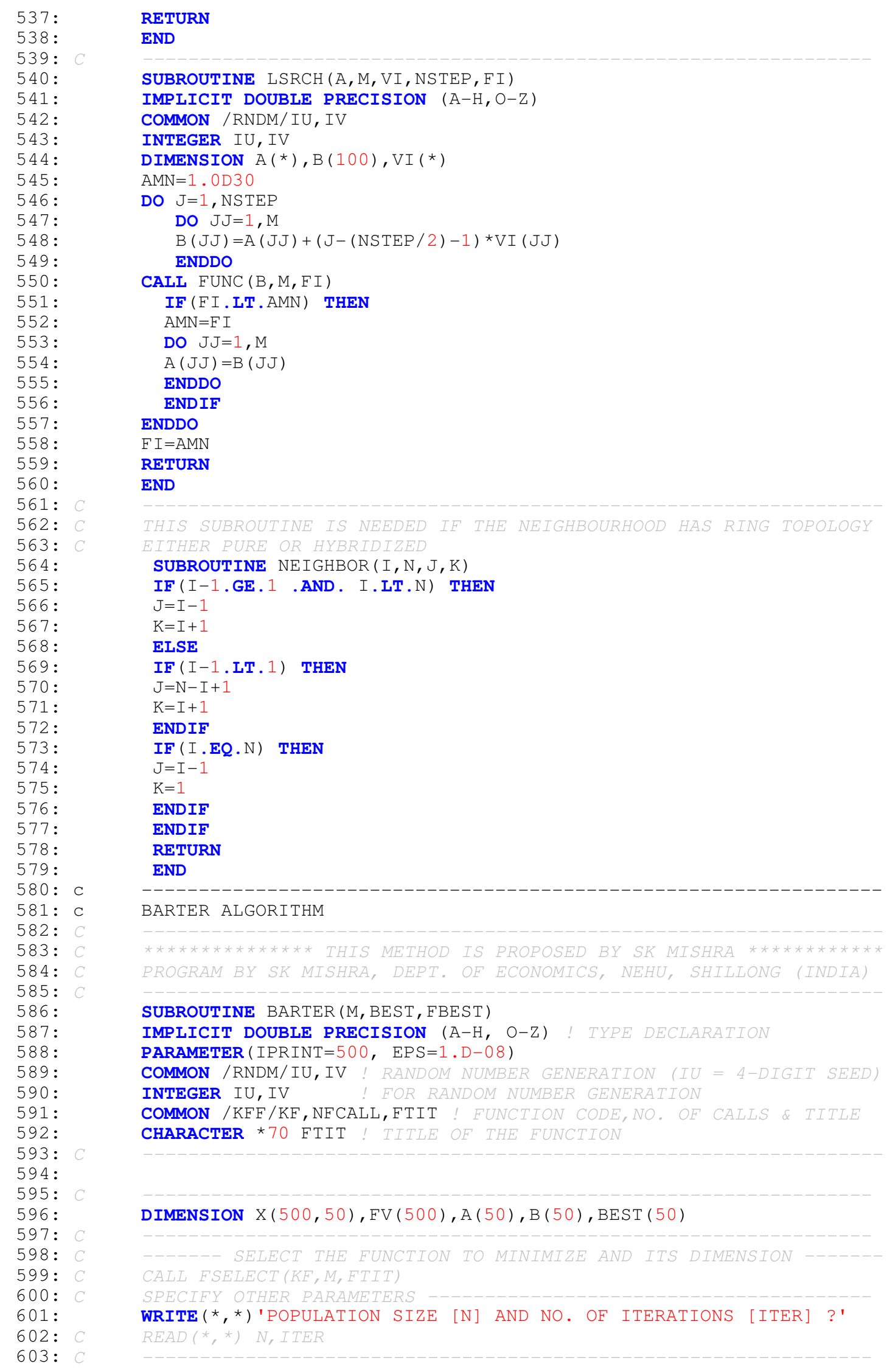




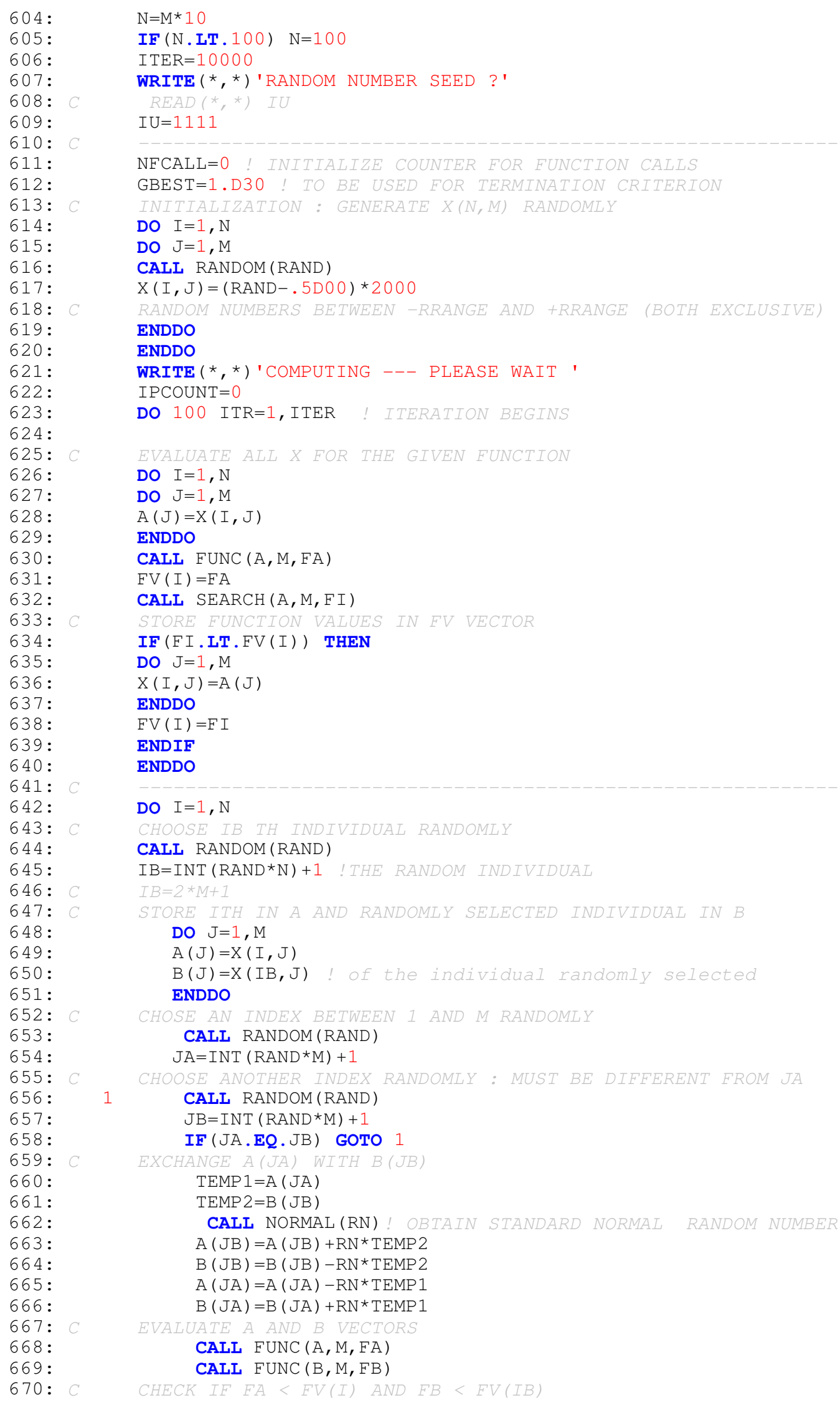




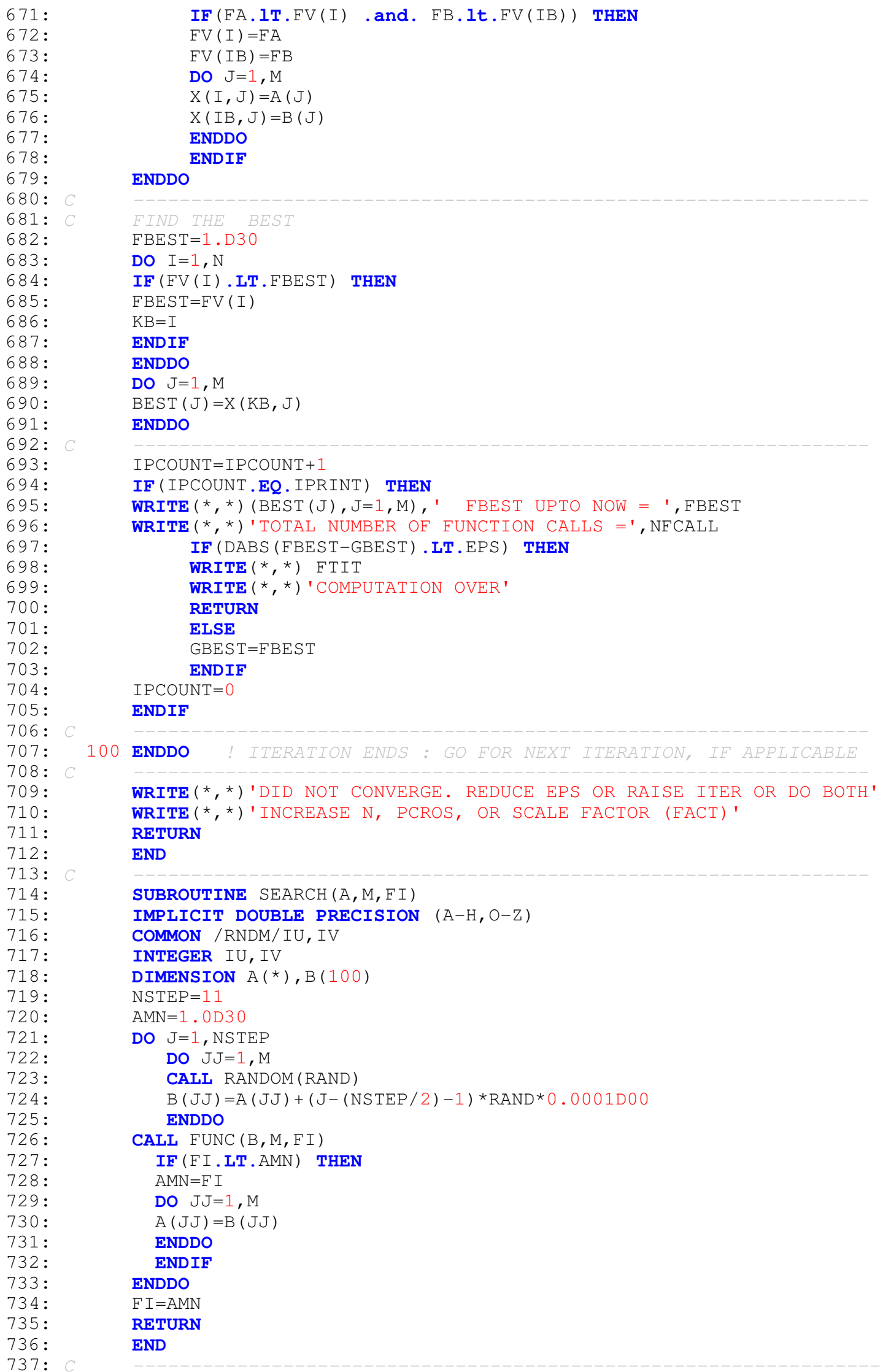


$738:$

739 :

740 :

741 :

742 :

743 :

$744:$

$745:$

$746:$

$747:$

748 :

$749:$

750 :

$751:$

$752:$

$753:$

$754:$

$755:$

756 :

$757:$

$758:$

759 :

760 :

$761:$

$762:$

763 :

$764:$

$765:$

$766:$

$767:$

768 :

769 :

$770:$

$771:$

$772:$

$773:$

774 :

$775:$

776 :

$777:$

778 :

$779:$

780 :

781 :

782 :

$783:$

$784:$

785 :

786 :

787 :

788 :

789 :

790 :

791 :

792 :

793 :

794 :

795 :

796 :

797:

798:

799 :

800 :

801 :

802 :

803:

804 :
SUBROUTINE FSELECT (KF, M,FTIT)

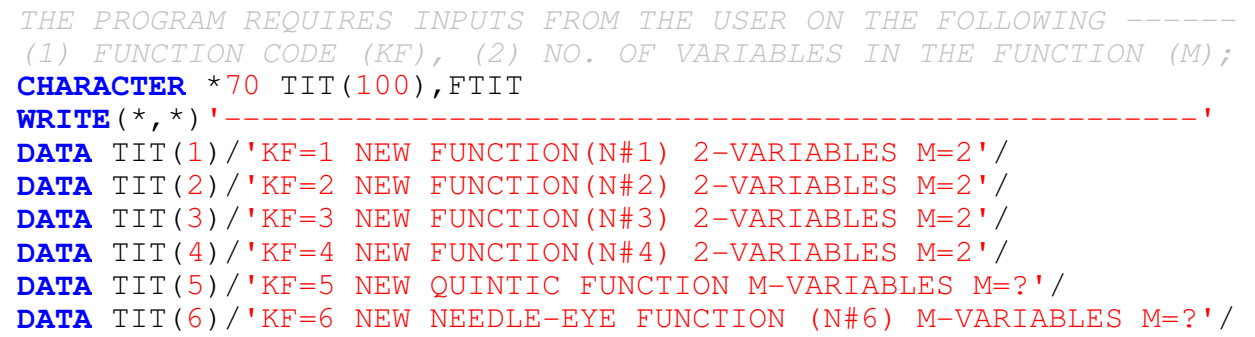

DATA TIT (7)/'KF=7 NEW ZERO-SUM FUNCTION (N\#7) M-VARIABLES M=?'/

DATA TIT (8)/'KF=8 CORANA FUNCTION 4-VARIABLES M=4'/

DATA TIT(9)/'KF=9 MODIFIED RCOS FUNCTION 2-VARIABLES M=2'/

DATA TIT(10)/'KF=10 FREUDENSTEIN ROTH FUNCTION 2-VARIABLES M=2'/

DATA TIT(11)/'KF=11 ANNS XOR FUNCTION 9-VARIABLES M=9'/

DATA TIT(12)/'KF=12 PERM FUNCTION \#1 (SET BETA) 4-VARIABLES M=4'/

DATA TIT(13)/'KF=13 PERM FUNCTION \#2 (SET BETA) M-VARIABLES M=?'/

DATA TIT(14)/'KE=14 POWER-SUM FUNCTION 4-VARIABLES M=4'/

DATA TIT(15)/'KF=15 GOLDSTEIN PRICE FUNCTION 2-VARIABLES M=2'/

DATA TIT(16)/'KF=16 BUKIN 6TH FUNCTION 2-VARIABLES M=2'/

DATA TIT (17)/'KF=17 NEW FUNCTION (N\#8) 2-VARIABLES M=2 '/

DATA TIT(18)/'KF=18 DEFL CORRUG SPRING FUNCTION M-VARIABLES M=?'/

DATA TIT(19)/'KF=19 NEW FACTORIAL FUNCTION M-VARIABLES M=?'/

DATA TIT (20)/'KF=20 NEW DECANOMIAL FUNCTION 2-VARIABLES M=2'/

DATA TIT(21)/'KF=21 JUDGE FUNCTION 2-VARIABLES M=2' /

DATA TIT (22)/'KF=22 NEW DODECAL FUNCTION 3-VARIABLES M=3'/

DATA TIT(23)/'KF=23 NEW SUM-EQ-PROD FUNCTION 2-VARIABLES M=2' /

DATA TIT (24)/'KF=24 NEW AM-EQ-GM FUNCTION M-VARIABLES M=?' /

DATA TIT(25)/'KF=25 YAO-LIU FUNCTION\#2 M-VARIABLES M=?'/

DATA TIT (26)/'KF=26 YAO-LIU FUNCTION\#3 M-VARIABLES M=?'/

DATA TIT (27)/'KF=27 YAO-LIU FUNCTION\#4 M-VARIABLES M=?'/

DATA TIT $(28) / / \mathrm{KF}=28$ YAO-LIU FUNCTION\#6 M-VARIABLES M=?'/

DATA TIT(29)/'KF=29 YAO-LIU FUNCTION\#7 M-VARIABLES M=?'/

DATA TIT(30)/'KF=30 YAO-LIU FUNCTION\#12 M-VARIABLES M=?'/

DATA TIT(31)/'KF=31 YAO-LIU FUNCTION\#13 M-VARIABLES M=?'/

DATA TIT(32)/'KF=32 YAO-LIU FUNCTION\#14 2-VARIABLES M=2'/

DATA TIT(33)/'KF=33 YAO-LIU FUNCTION\#15 4-VARIABLES M=4'/

DATA TIT(34)/'KF=34 WOOD FUNCTION : 4-VARIABLES M=4'/

DATA TIT (35)/'KF=35 FENTON-EASON FUNCTION : 2-VARIABLES M=2'/

DATA TIT(36)/'KF=36 HOUGEN FUNCTION : 5-VARIABLES M=5 /

DATA TIT(37)/'KF=37 GIUNTA FUNCTION : 2-VARIABLES M=2'/

DATA TIT(38)/'KF=38 EGGHOLDER FUNCTION : M-VARIABLES M=?'/

DATA TIT(39)/'KF=39 TRID FUNCTION : M-VARIABLES M=?'/

DATA TIT (40)/'KF=40 GRIEWANK FUNCTION : M-VARIABLES M=?'/

DATA TIT $(41) / / K F=41$ WEIERSTRASS FUNCTION : M-VARIABLES M=?'/

DATA TIT(42)/'KF=42 LEVY-3 FUNCTION : 2-VARIABLES M=2'/

DATA TIT (43)/'KF=43 LEVY-5 FUNCTION : 2-VARIABLES M=2'/

DATA TIT (44)/'KF=44 LEVY-8 FUNCTION : 3-VARIABLES M=3'/

DATA TIT $(45) /$ 'KF=45 RASTRIGIN FUNCTION : M-VARIABLES M=?'/

DATA TIT (46)/'KF=46 ACKLEY FUNCTION : M-VARIABLES M=?' /

DATA TIT (47)/'KF=47 MICHALEWICZ FUNCTION : M-VARIABLES M=?' /

DATA TIT (48)/'KF=48 SCHWEFEL FUNCTION : M-VARIABLES M=?'/

DATA TIT(49)/'KF=49 SHUBERT FUNCTION : 2-VARIABLES M=2'/

DATA TIT (50)/'KF=50 DIXON-PRICE FUNCTION : M-VARIABLES M=?'/

DATA TIT(51)/'KE=51 SHEKEL FUNCTION : 4-VARIABLES M=4'/

DATA TIT (52)/'KF=52 PAVIANI FUNCTION : 10-VARIABLES M=10 /

DATA TIT(53)/'KF=53 BRANIN FUNCTION\#1 : 2-VARIABLES M=2'/

DATA TIT(54)/'KF=54 BRANIN FUNCTION\#2 : 2-VARIABLES M=2'/

DATA TIT (55)/'KE=55 BOHACHEVSKY FUNCTION\#1 : 2-VARIABLES M=2'/

DATA TIT (56)/'KF=56 BOHACHEVSKY FUNCTION\#2: 2-VARIABLES M=2'/

DATA TIT (57)/'KF=57 BOHACHEVSKY FUNCTION\#3 : 2-VARIABLES M=2' /

DATA TIT (58)/'KF=58 EASOM FUNCTION : 2-VARIABLES M=2'/

DATA TIT(59)/'KF=59 ROSENBROCK FUNCTION : M-VARIABLES M=?'/

DATA TIT(60)/'KF=60 CROSS-LEGGED TABLE FUNCTION:2-VARIABLES M=2 '/

DATA TIT(61)/'KF=61 CROSS FUNCTION : 2-VARIABLES M=2'/

DATA TIT(62)/'KF=62 CROSS-IN-TRAY FUNCTION : 2-VARIABLES M=2'/ 


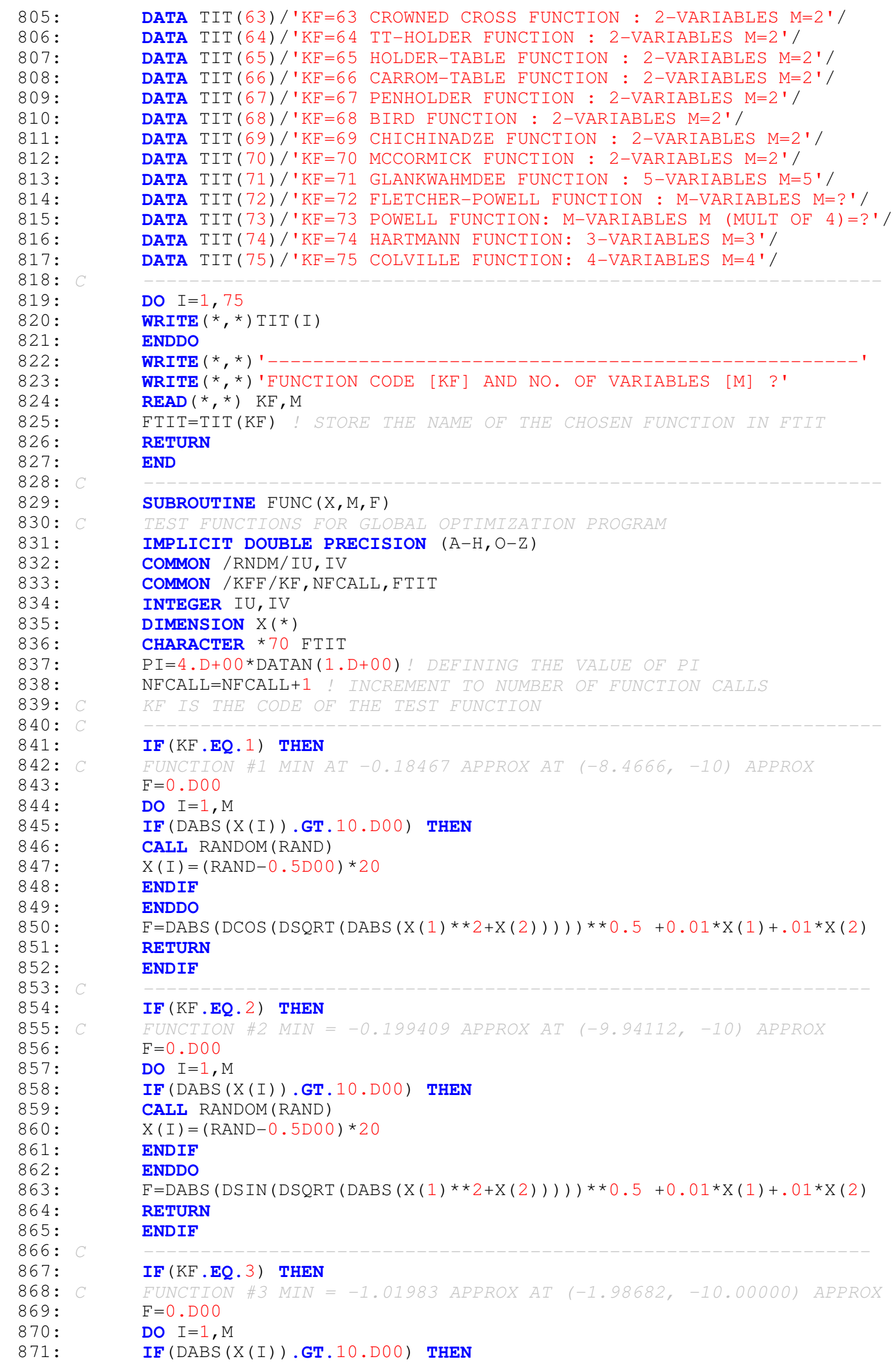




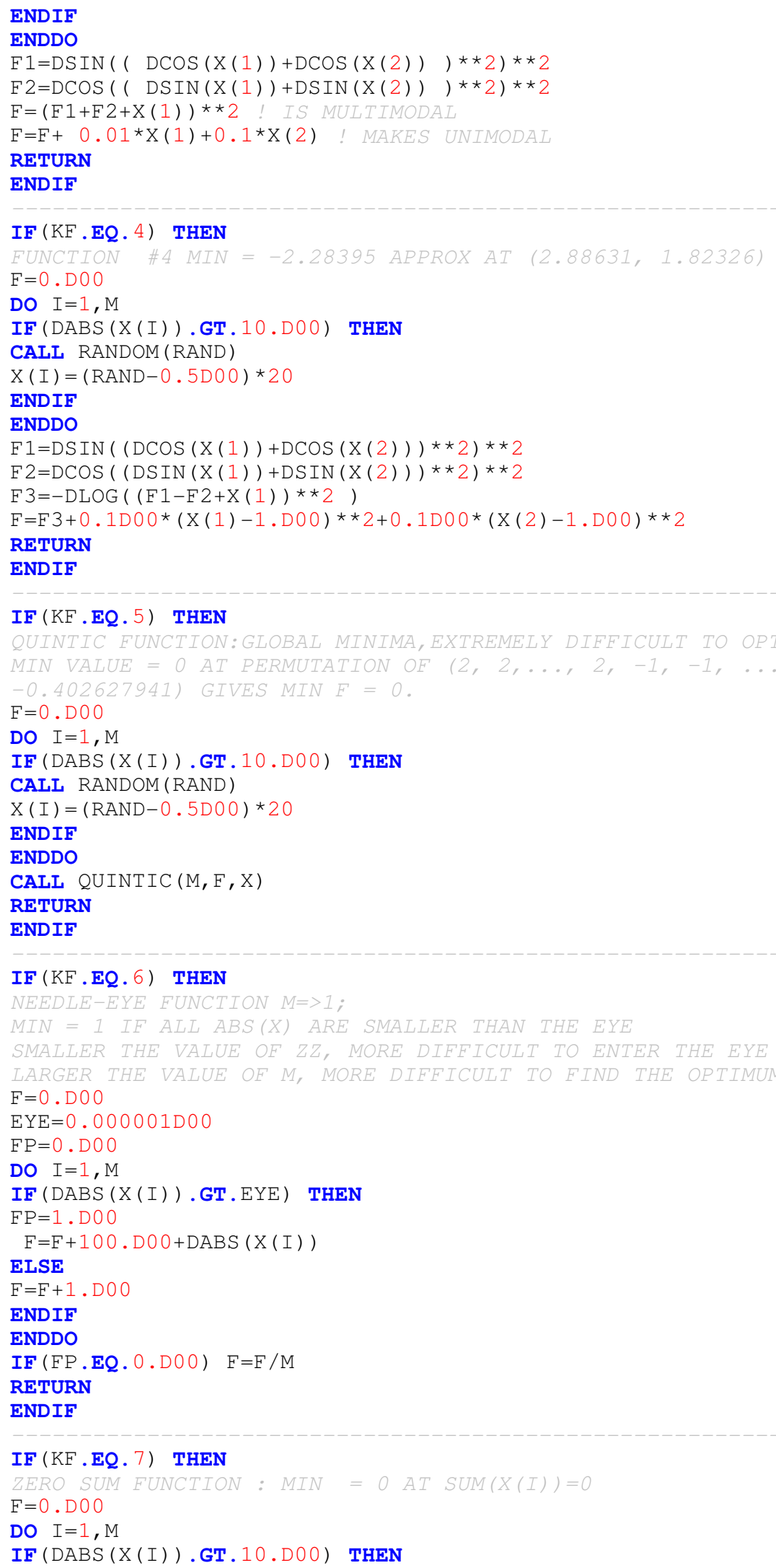




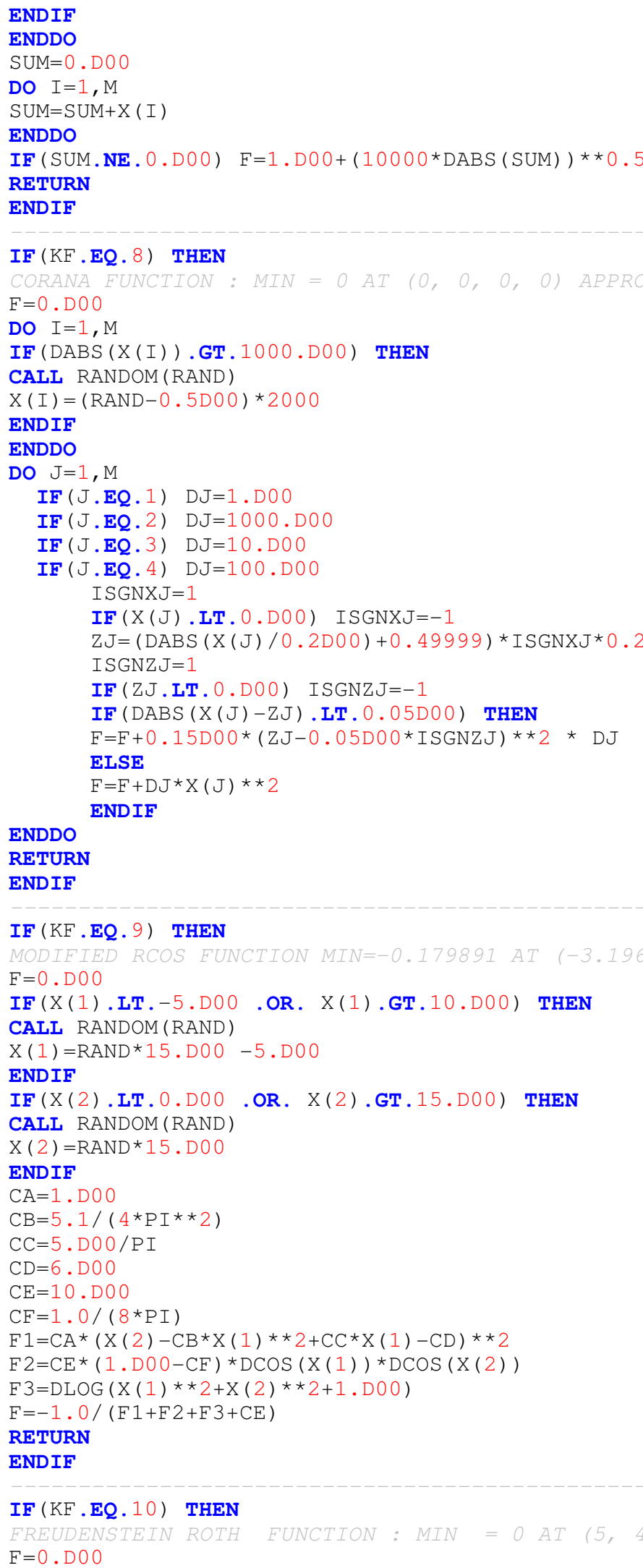




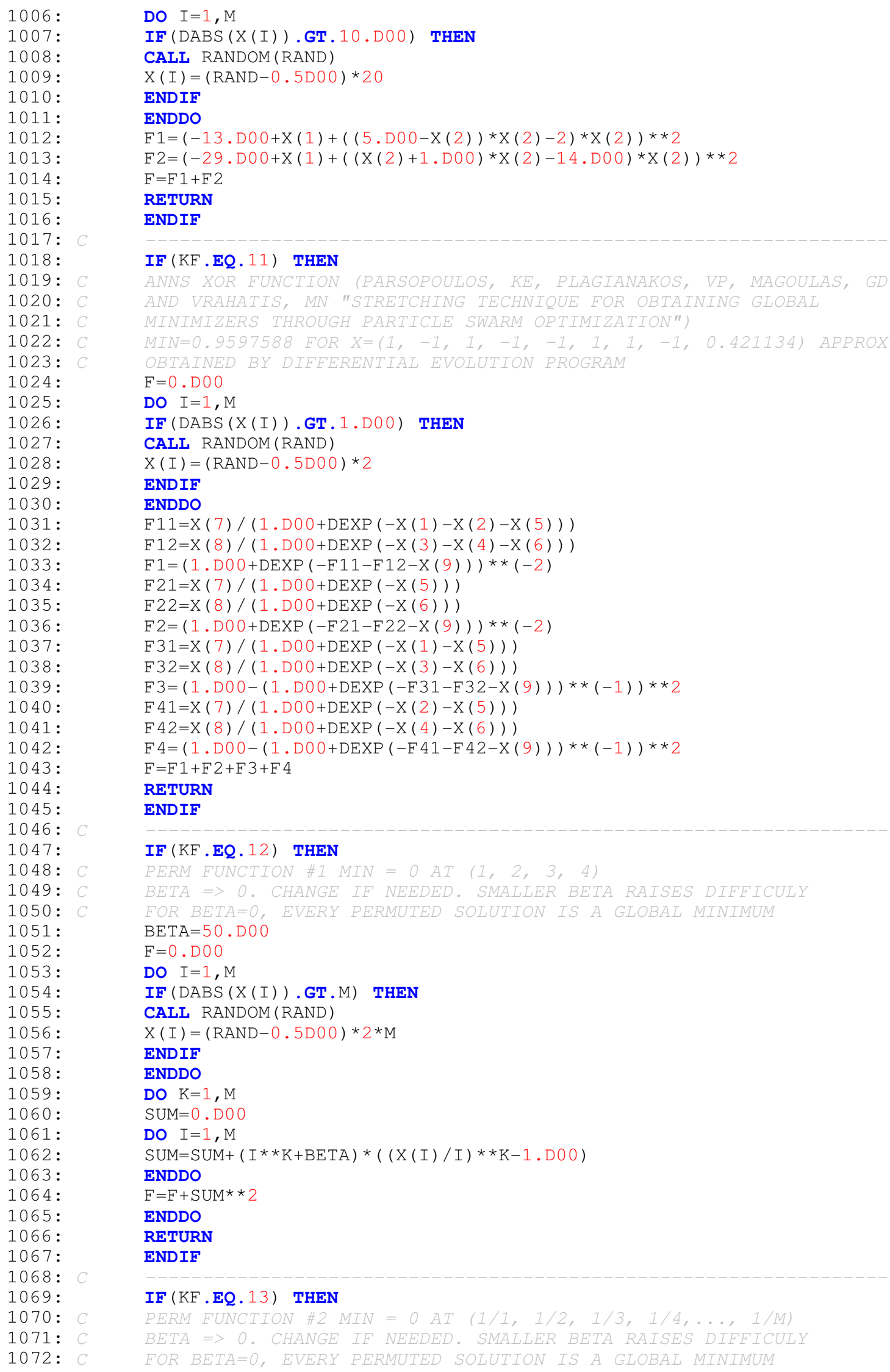




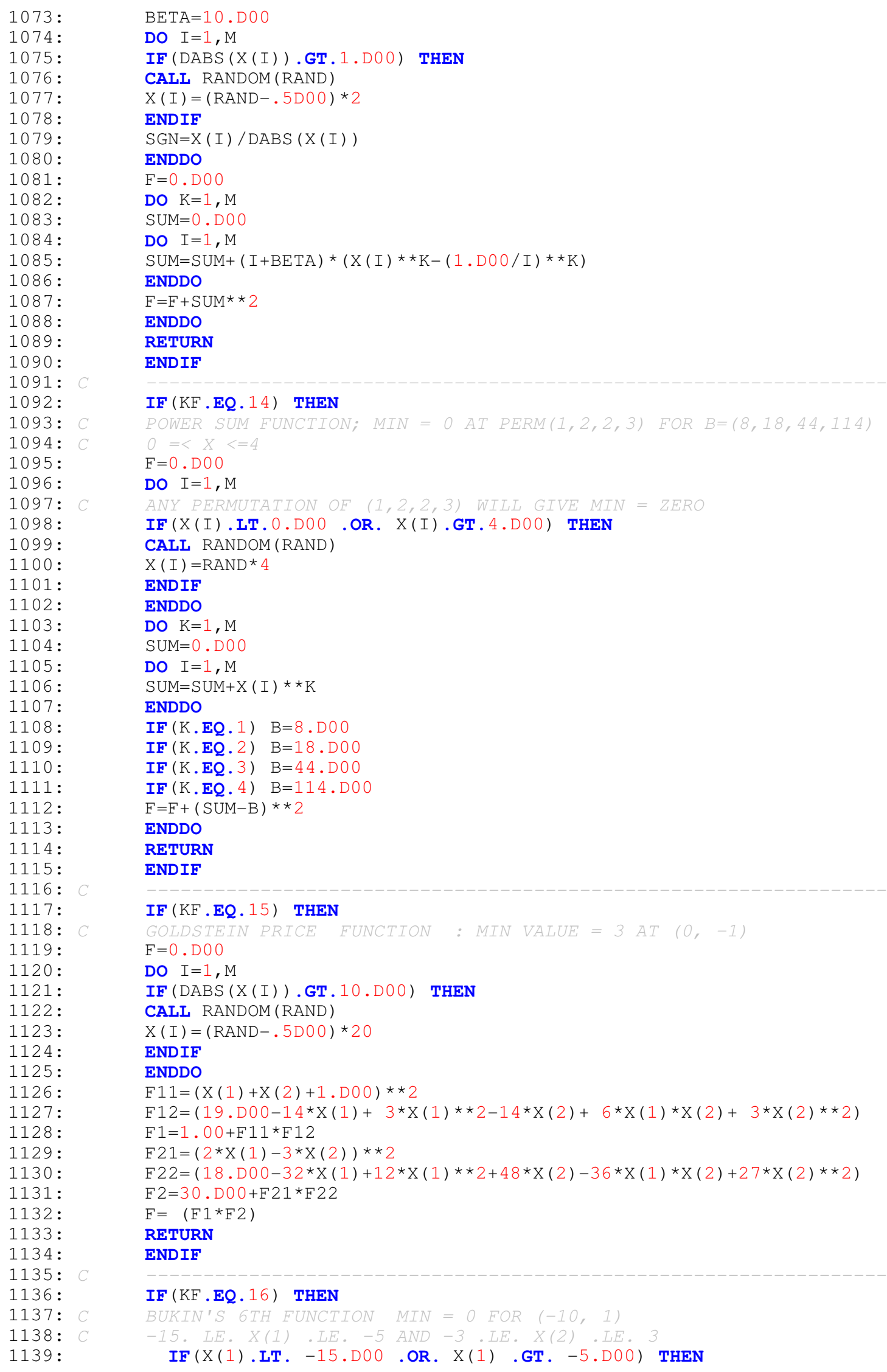




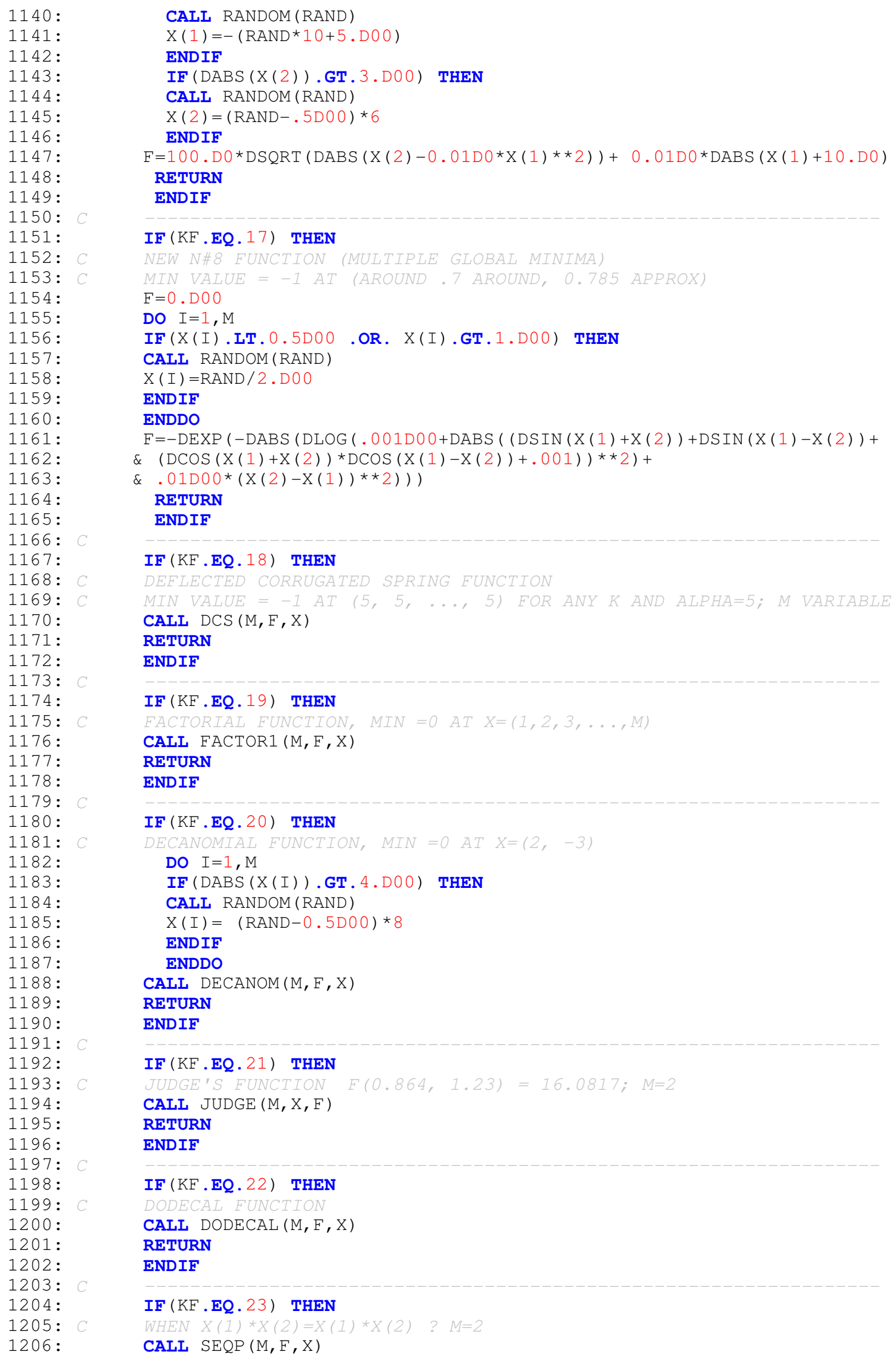




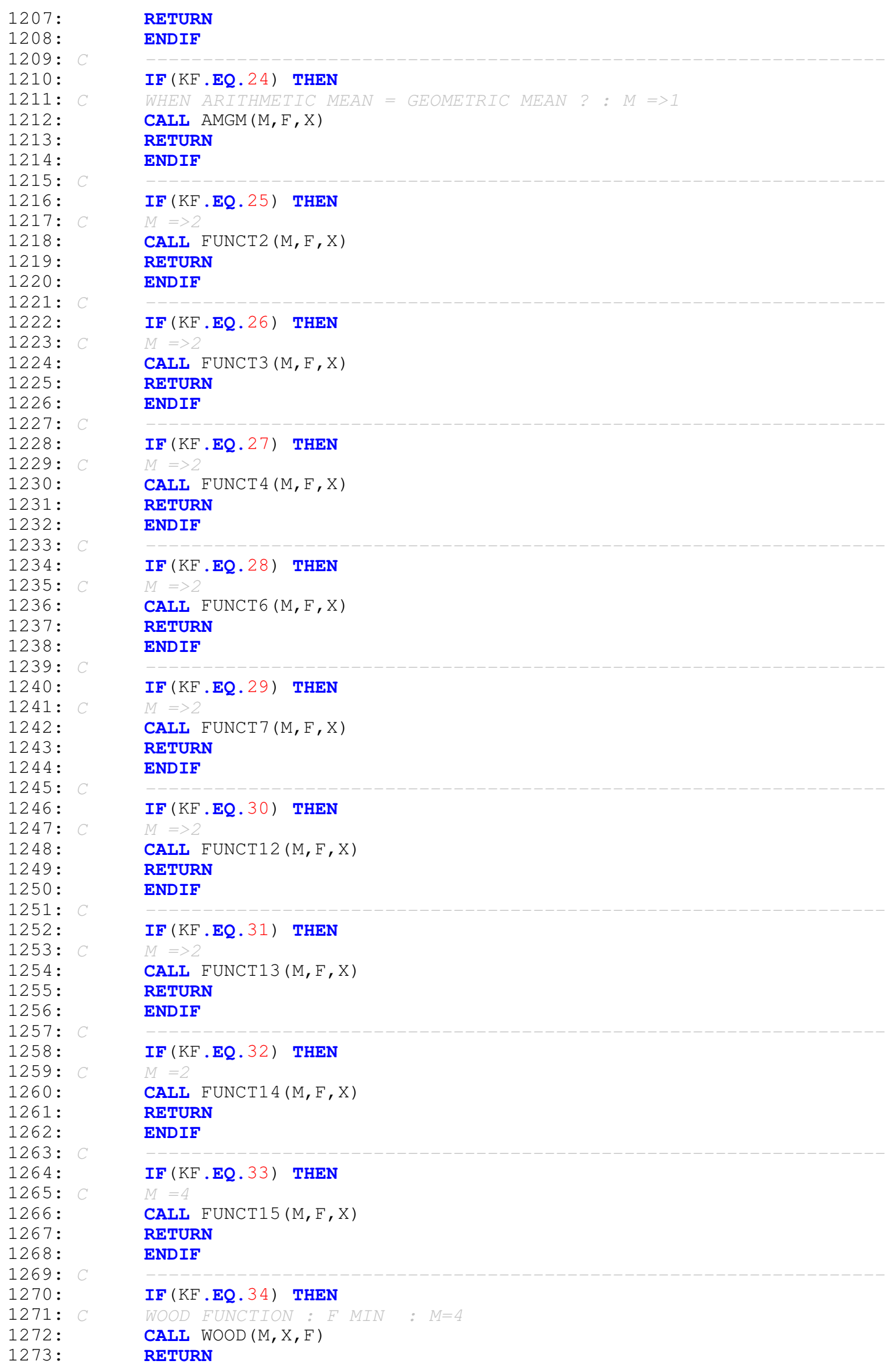




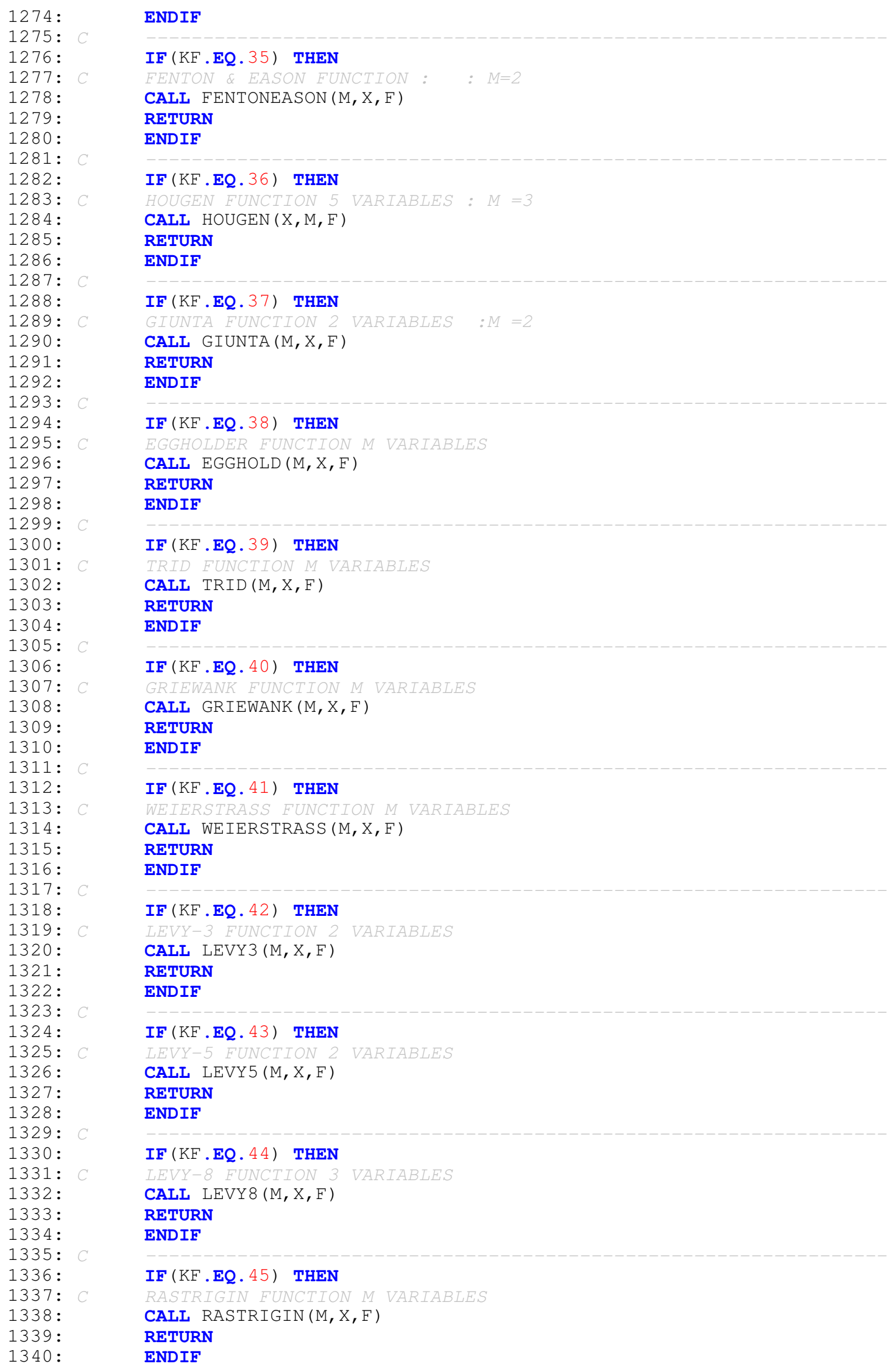


1341:

1342:

1343:

1344:

1345:

1346:

1347:

1348:

1349:

1350:

1351:

1352:

1353:

1354:

1355:

1356:

1357:

1358:

1359:

1360:

1361:

1362:

1363:

$1364:$

1365:

1366 :

1367:

1368:

1369:

1370 :

1371:

1372:

1373:

1374:

1375:

1376:

1377:

1378:

1379:

1380:

1381:

1382:

1383:

1384 :

1385:

1386:

1387:

1388:

1389:

1390 :

1391:

1392:

1393:

1394:

1395:

1396 :

1397:

1398:

1399:

1400:

1401:

1402:

1403:

1404:

1405:

1406:

1407:
IF (KF.EQ. 46) THEN

CALL ACKLEY $(\mathrm{M}, \mathrm{X}, \mathrm{F})$

RETURN

ENDIF

IF (KF.EQ. 47) THEN

CALL MICHALEWICZ (M, X, F)

RETURN

ENDIF

IF (KF.EQ. 48) THEN

SCHWEFEI FUNCTION M VARIABLES

CALL SCHWEFEL (M, X, F)

RETURN

ENDIF

IF (KF.EQ. 49) THEN

SHUBERT FUNCTION 2 VARIABLES

CALL SHUBERT $(M, X, F)$

RETURN

ENDIF

IF (KF . EQ. 50) THEN

CALL DIXPRICE $(M, X, F)$

RETURN

ENDIF

IF (KF . EQ.51) THEN

CALI SHEKEL $(\mathrm{M}, \mathrm{X}, \mathrm{F})$

RETURN

ENDIF

IF (KF.EQ.52) THEN

PAVIANI FUNCIION 10 VARIABLES

CALL PAVIANI (M, X, F)

RETURN

ENDIF

IF (KF.EQ.53) THEN

BRANIN FUNCTION\#I 2 VARIABIES

CALL BRANIN1 $(M, X, F)$

RETURN

ENDIF

IF (KF.EQ. 54) THEN

BRANIN FUNCTION\#2 2

RETURN

ENDIF

IF (KF.EQ. 55) THEN

CALL BOHACHEVSKYI (M, X, F)

RETURN

ENDIF

IF (KF.EQ. 56) THEN

CALL BOHACHEVSKY2 $(M, X, F)$

RETURN

ENDIF 


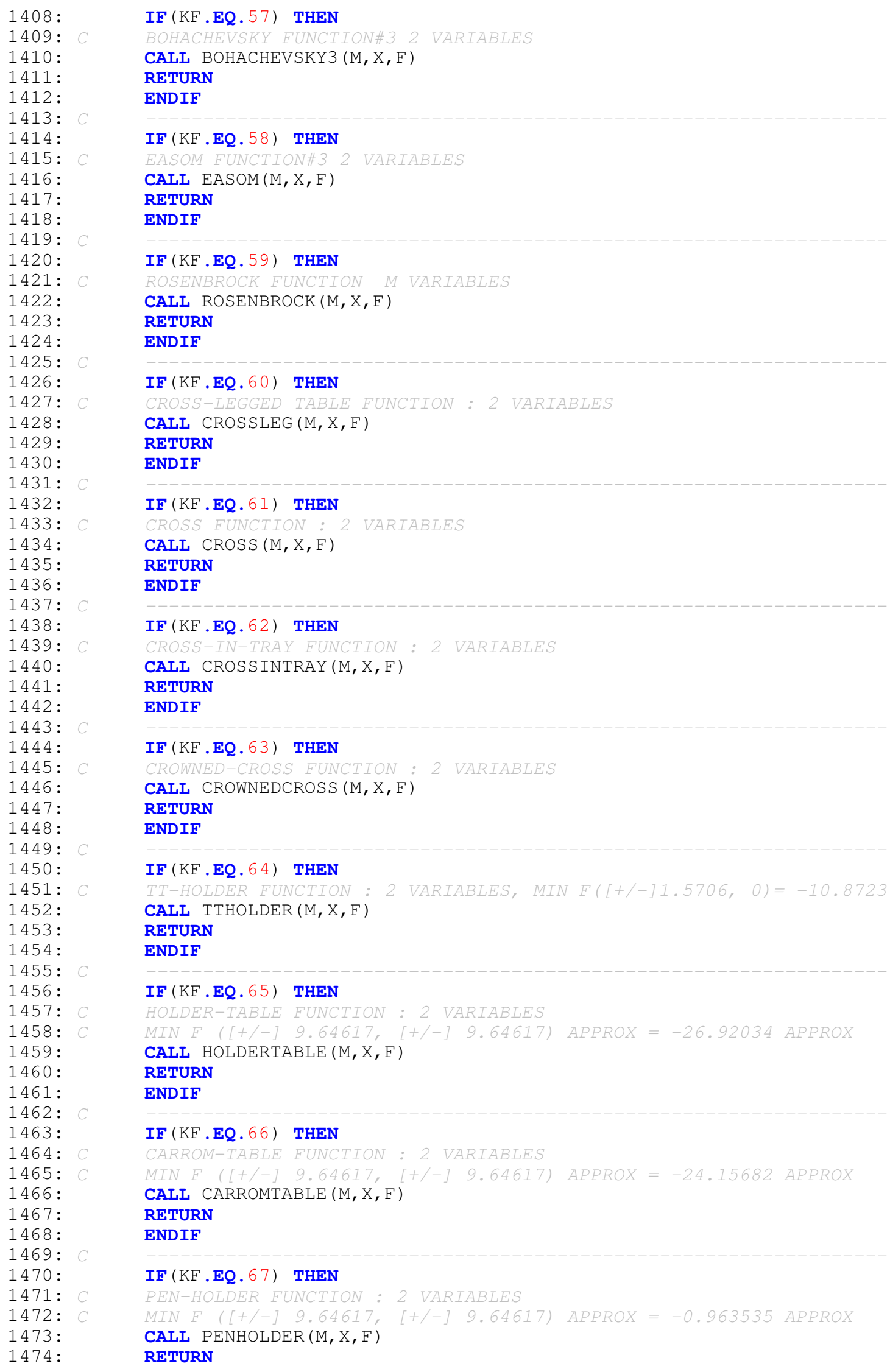




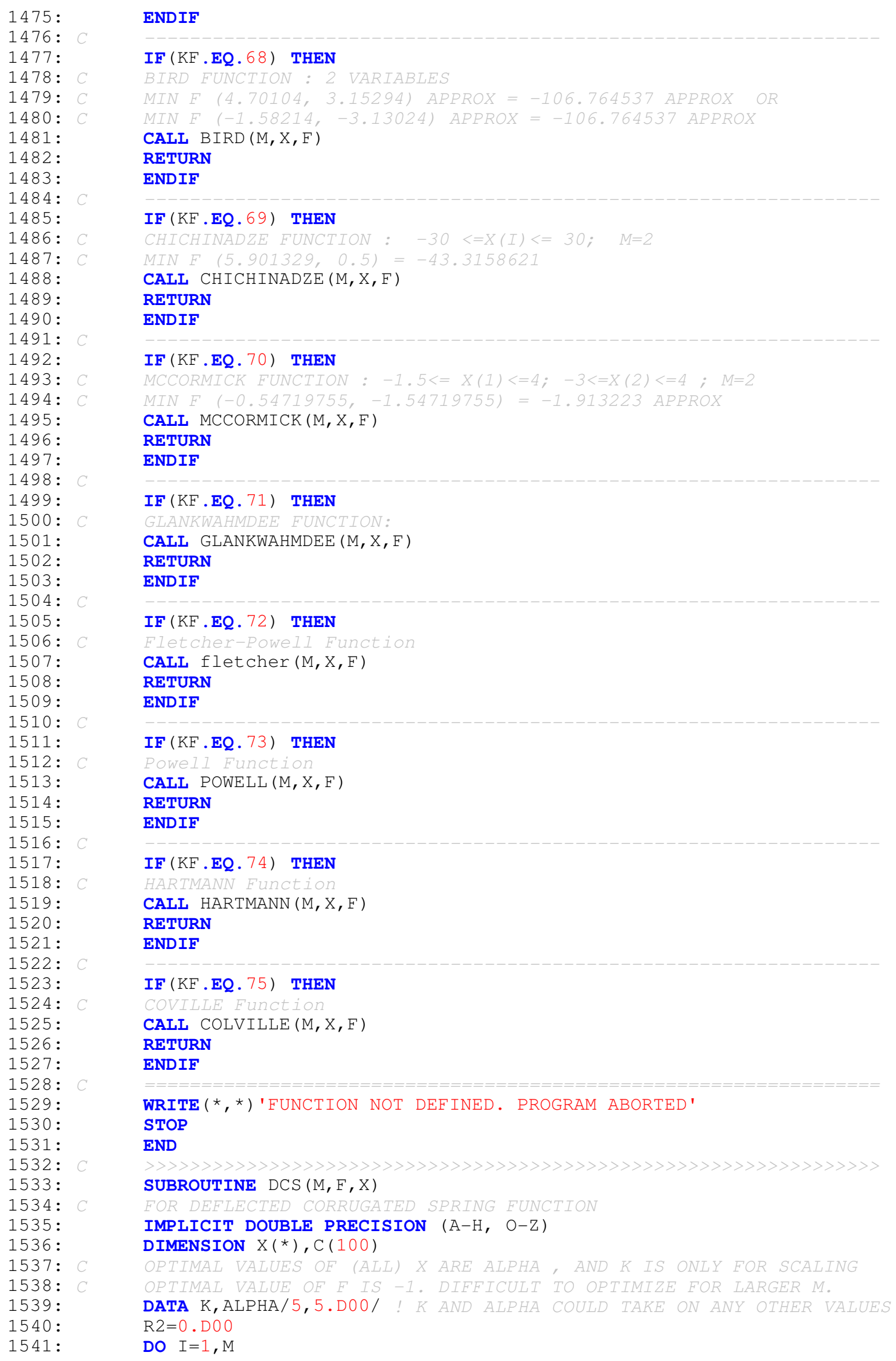




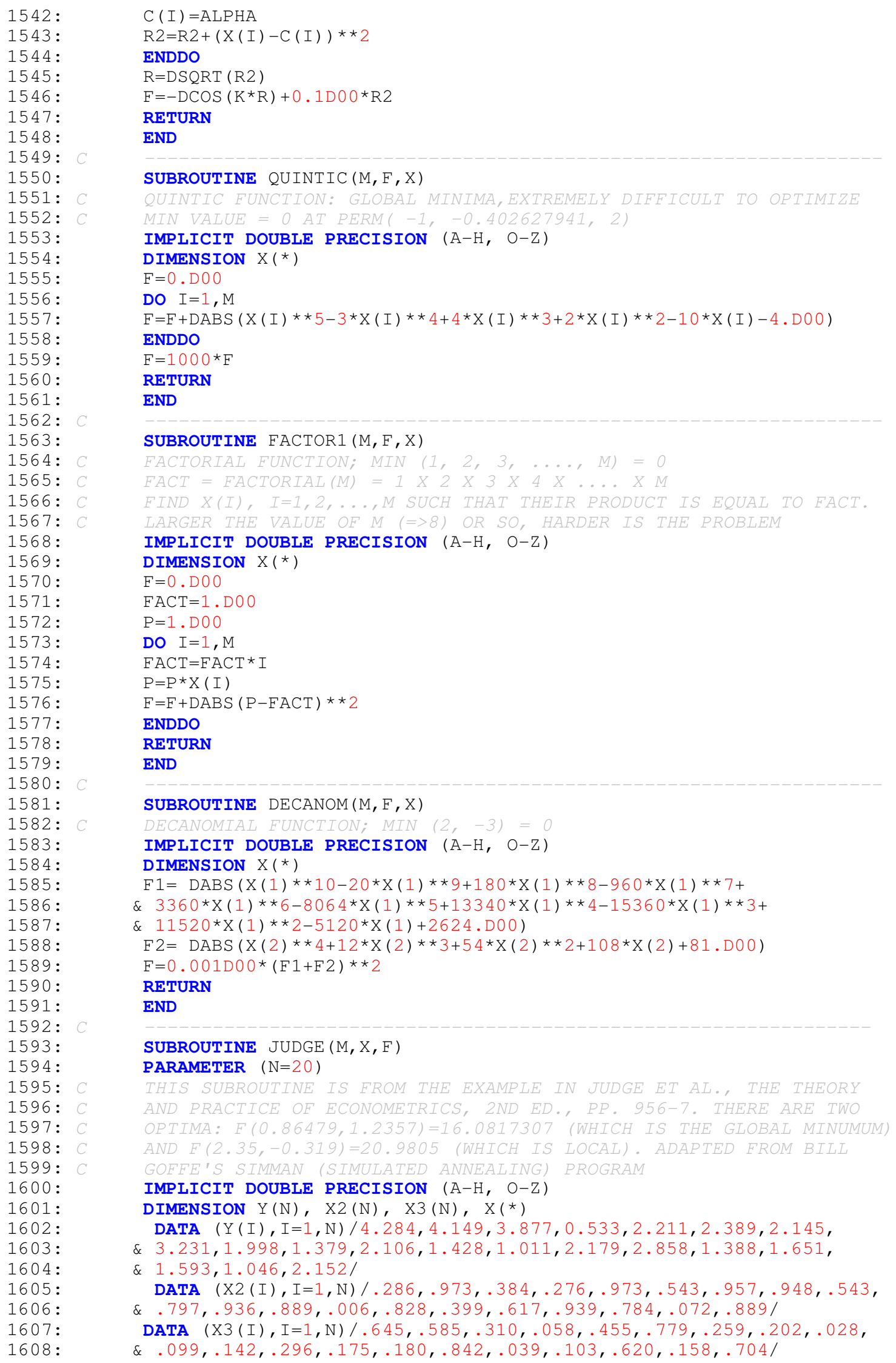




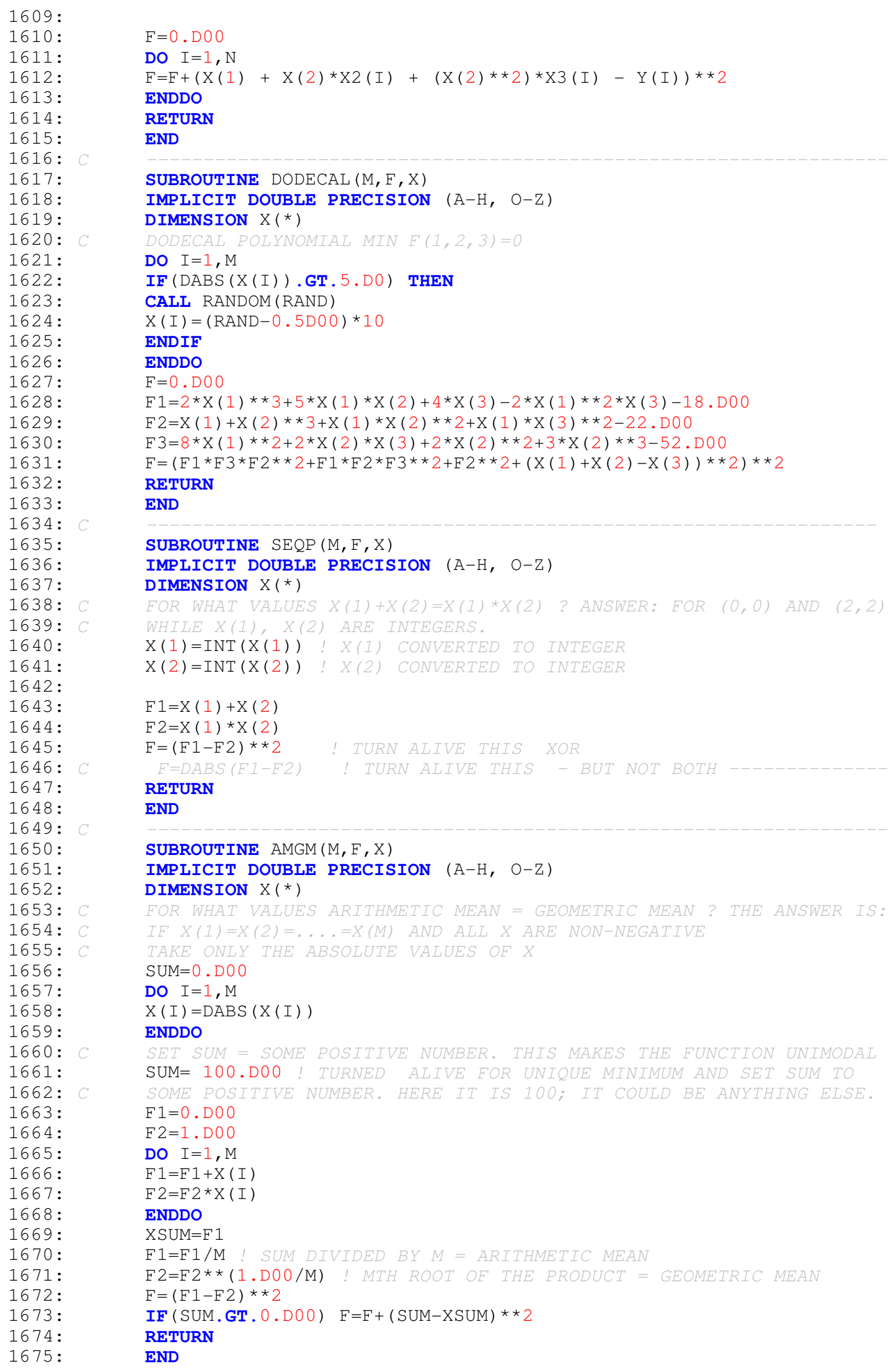


1676:

1677:

1678:

1679:

1680:

1681:

1682:

1683:

1684:

1685:

1686 :

1687:

1688:

1689:

1690:

1691:

1692:

$1693:$

1694:

1695:

$1696:$

1697:

1698:

1699:

1700:

1701:

1702:

1703:

1704:

1705:

1706:

1707:

1708:

1709:

1710:

1711:

1712:

1713:

1714:

1715:

1716:

1717:

1718:

1719:

1720:

1721:

1722:

1723:

1724:

1725:

1726:

1727:

1728:

1729:

1730:

1731:

1732:

1733:

1734:

1735:

1736:

1737:

1738:

1739:

1740 :

1741:

1742:

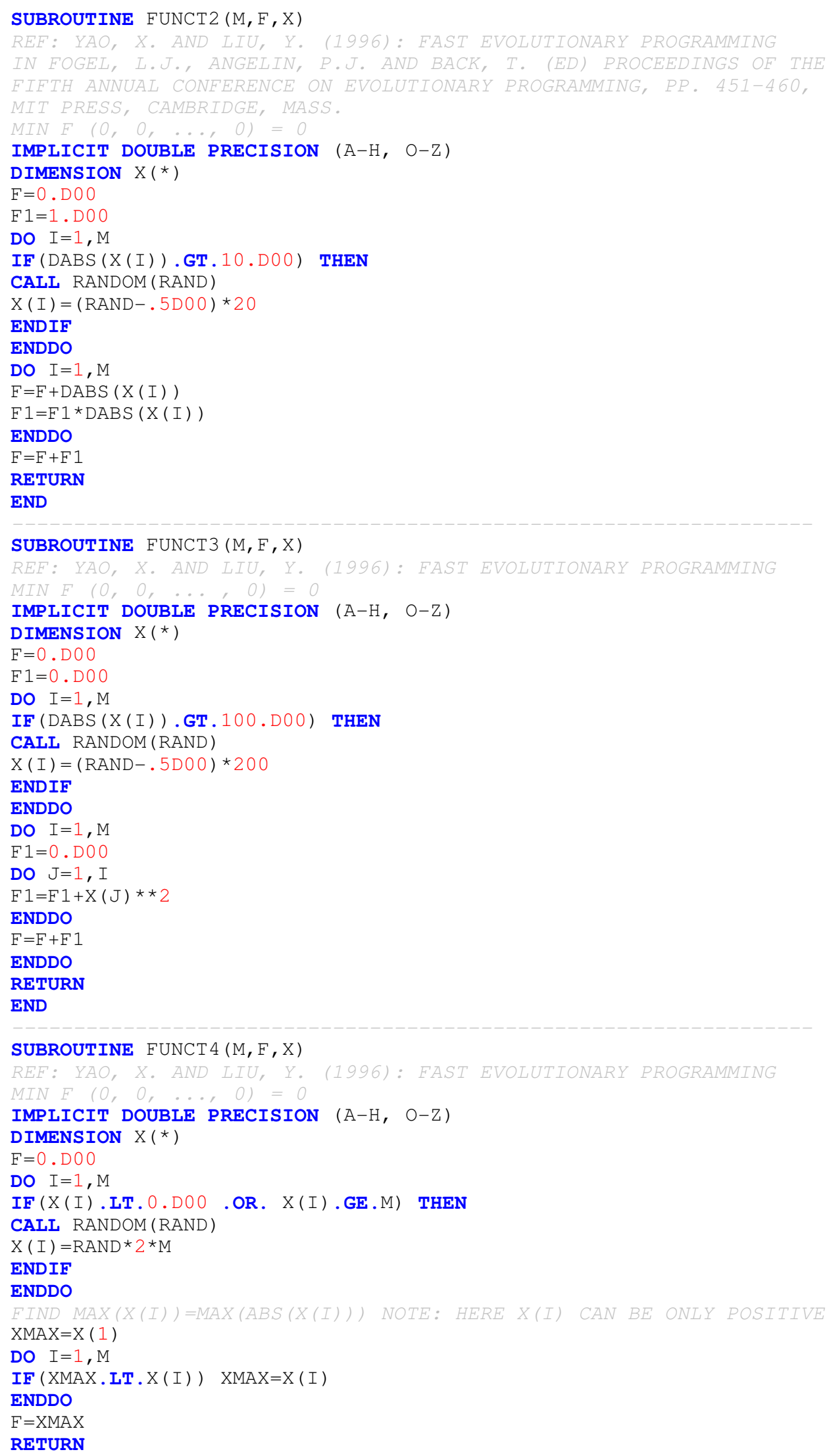




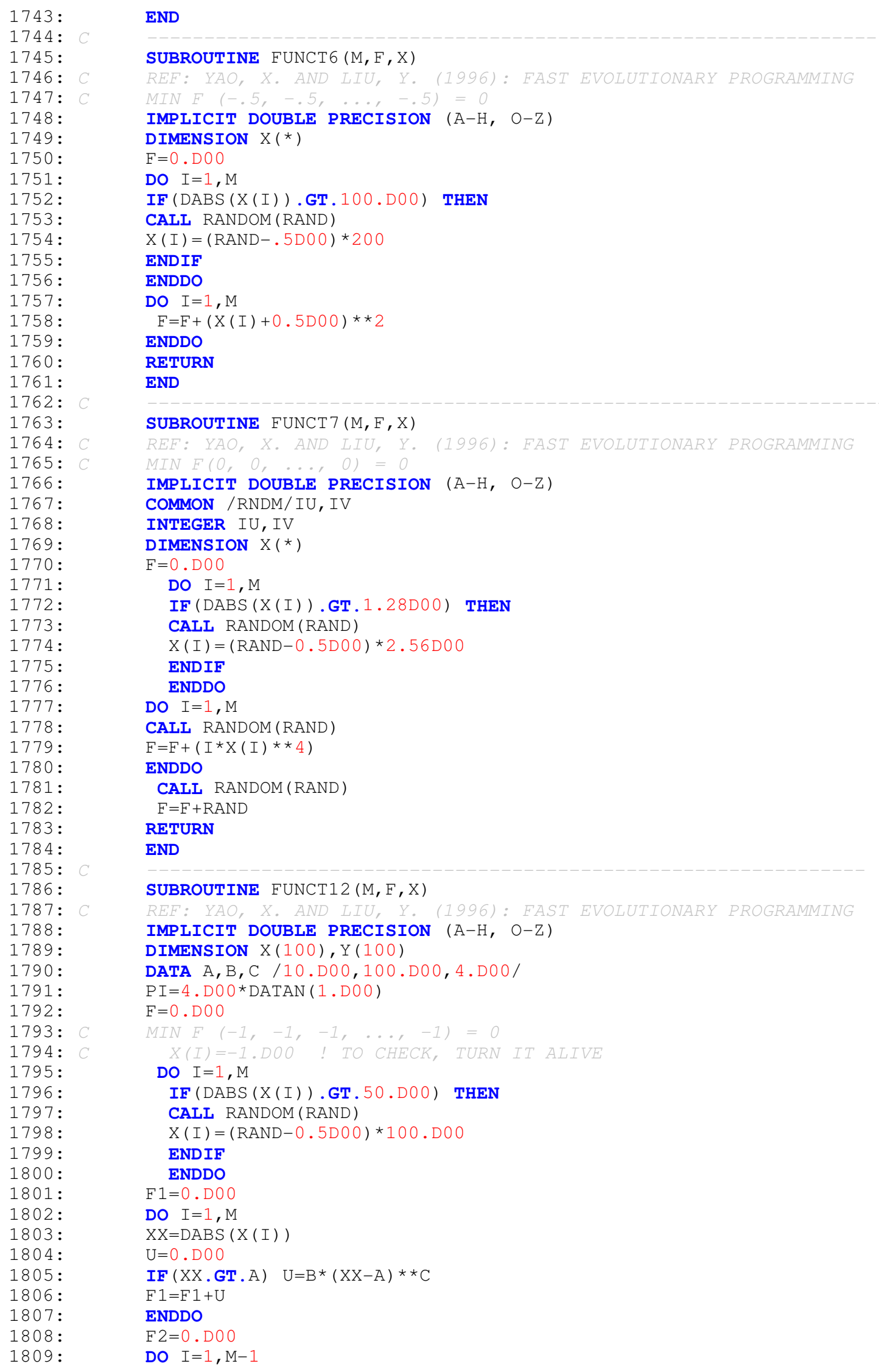




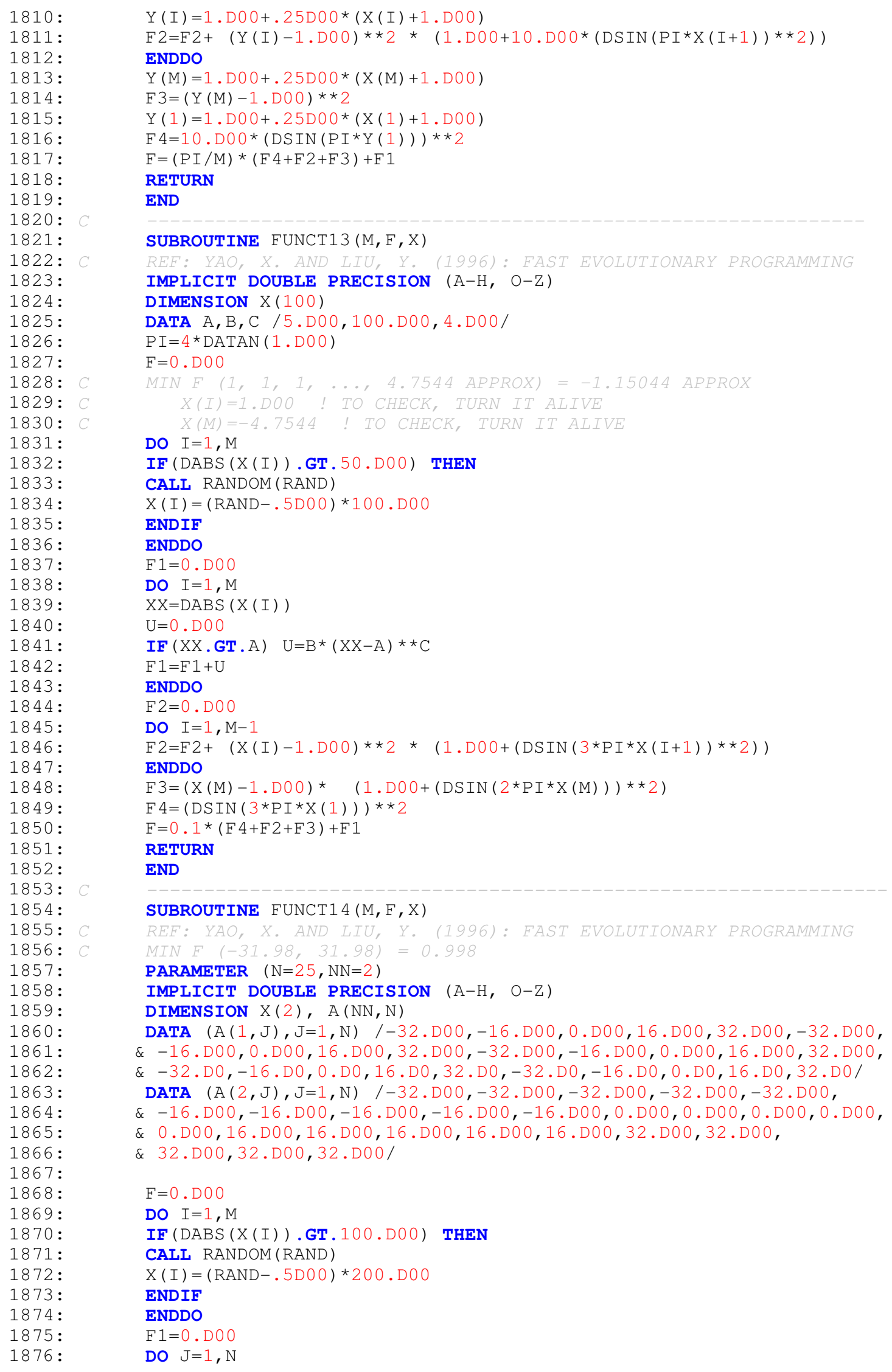




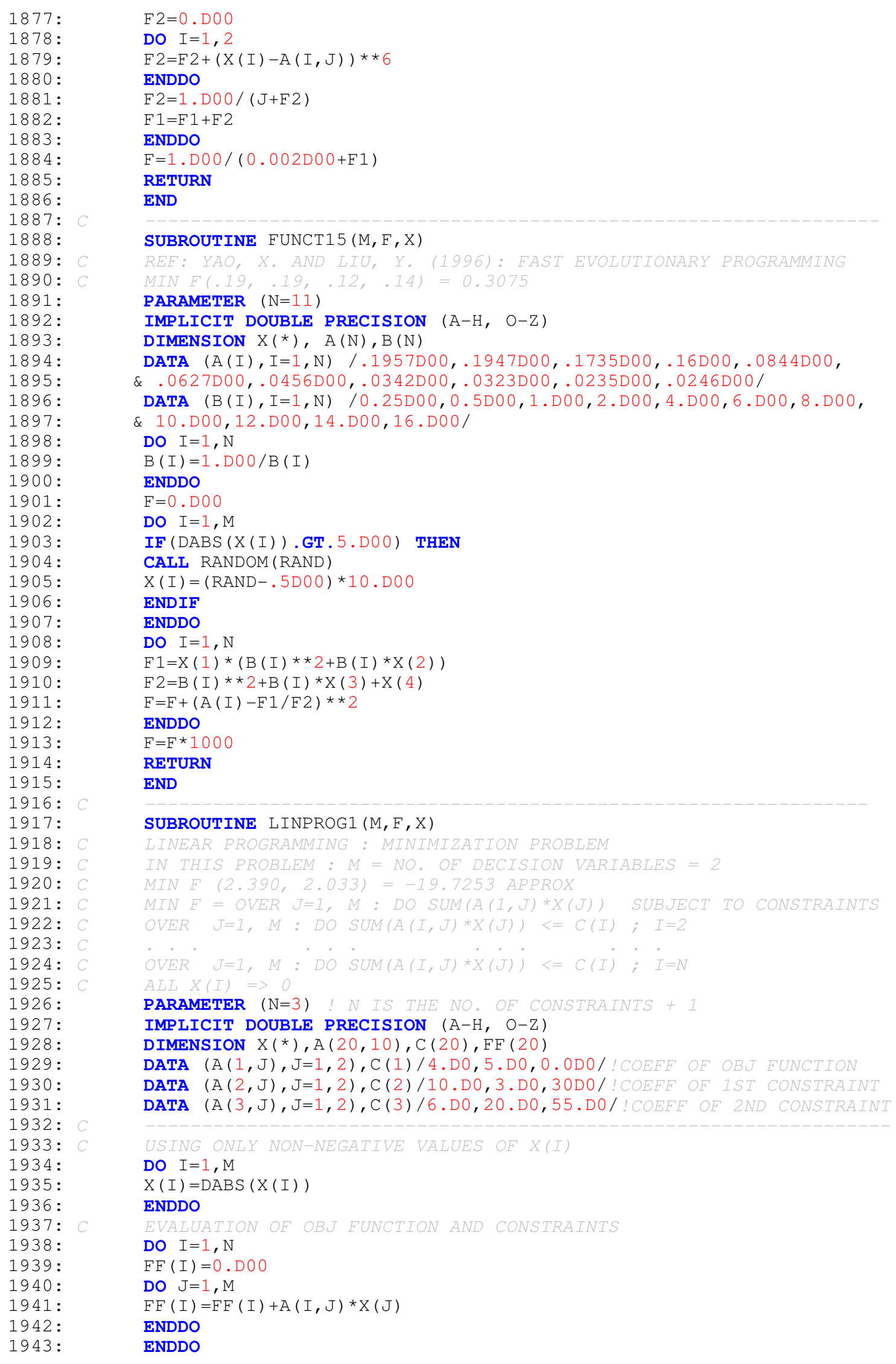




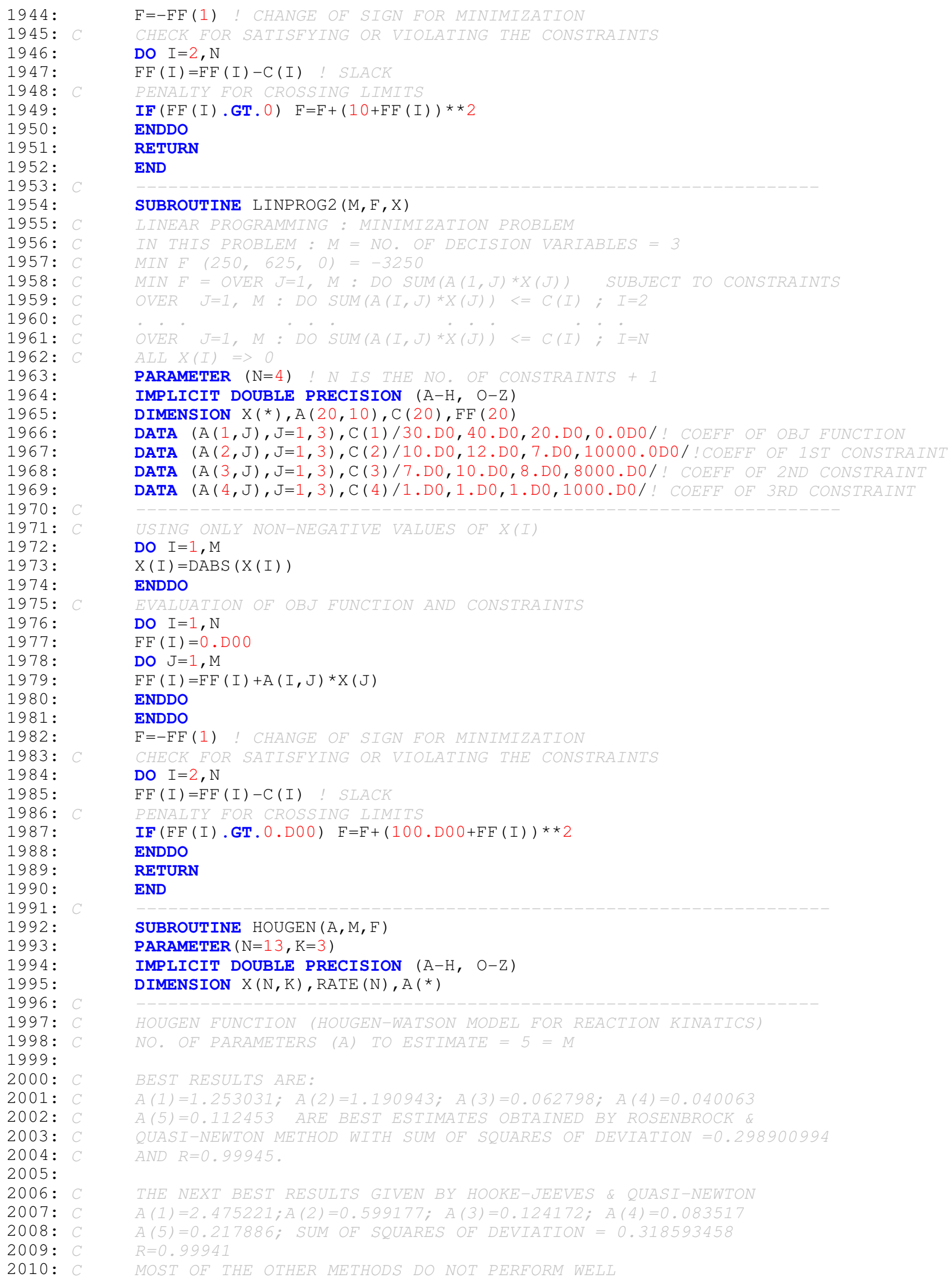

1944:

1945:

1946:

1947:

1948:

1949 :

1950:

1951:

1952:

1953:

1954:

1955:

1956:

1957:

1958:

1959:

1960:

1961:

1962:

1963:

1964:

1965:

1966:

1967:

1968:

1969:

1970:

1971:

1972:

1973:

1974:

$1975:$

1976:

1977:

1978:

1979:

1980 :

1981:

1982:

1983:

1984 :

1985:

1986:

1987:

1988:

1989:

1990:

1991:

1992:

1993:

1994:

1995:

1996:

1997:

1998:

1999:

2000:

2001:

2002:

2003:

2004:

2005:

2006:

2007:

2008:

2009:

2010:

$\mathrm{F}=-\mathrm{FF}(1)$ ! CHANGE OF SIGN FOR MINIMIZATION

CHECK FOR SATISFYING OR VIOLATING THE CONSTRAINTS

DO $I=2, N$

$\mathrm{FF}(\mathrm{I})=\mathrm{FF}(\mathrm{I})-\mathrm{C}(\mathrm{I}) \quad$ ! SLACK

PENALTY FOR CROSSING LIMITS

IF (FF (I) .GT.0) $\quad \mathrm{F}=\mathrm{F}+(10+\mathrm{FF}(\mathrm{I})) \star \star 2$

ENDDO

RETURN

END

SUBROUTINE LINPROG2 (M, F, X)

IINEAR PROGRAMMING : MINIMIZATION PROBLEM

IN THIS PROBLEM : $M=N O$. OF DECISION VARIABLES = 3

MIN F $(250,625,0)=-3250$

MIN $F=O V E R$ J=1, $M: D O \operatorname{SUM}(A(I, J) * X(J)) \quad \operatorname{SUBJECT}$ TO CONSTRAINTS

OVER J=1, M : DO SUM $(A(I, J) * X(J)) \quad<=C(I) ; I=2$

OVER J=I, M : DO SUM $(A(I, J) * X(J)) \quad<=C(I) ; I=N$

$A L \perp X(1)$

PARAMETER $(\mathrm{N}=4)$

IMPLICIT DOUBLE PRECISION (A-H, O-Z)

DIMENSION $\mathrm{X}(*), \mathrm{A}(20,10), \mathrm{C}(20), \mathrm{FF}(20)$

DATA $(A(1, J), J=1,3), C(1) / 30 . D 0,40 . D 0,20 . D 0,0.0 D 0 / ! C O E F F$ OF OBJ FUNCTION

DATA $(A(2, J), J=1,3), C(2) / 10 . D 0,12 . D 0,7 . D 0,10000.0 D 0 /$ ! COEFF OF IST CONSTRAINT

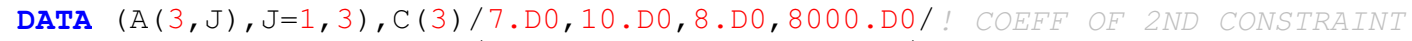

DATA (A (4,J), J=1,3),C(4)/1.D0,1.D0,1.D0,1000.D0/! COEFF OF 3RD CONSTRAINT

USING ONLY NON-NEGATIVE VALUES OF X(I)

DO $I=1, M$

$X(I)=\operatorname{DABS}(X(I))$

ENDDO

EVALUATION OF OBJ FUNCTION AND CONSTRAINTS

DO $I=1, N$

$\mathrm{FF}(\mathrm{I})=0 . \mathrm{D} 00$

DO $\mathrm{J}=1, \mathrm{M}$

$\mathrm{FF}(\mathrm{I})=\mathrm{FF}(\mathrm{I})+\mathrm{A}(\mathrm{I}, \mathrm{J}) * \mathrm{X}(\mathrm{J})$

ENDDO

ENDDO

$\mathrm{F}=-\mathrm{FF}(1)$ ! CHANGE OF SIGN FOR MINIMIZATION

CHECK EOR SATISEYING OR VIOIATING THE CONSTRAINTS

DO $I=2, N$

$\mathrm{FF}(\mathrm{I})=\mathrm{FF}(\mathrm{I})-\mathrm{C}(\mathrm{I}) \quad ! \quad S \mathrm{~S} A C K$

PENALTY FOR CROSSING LIMITS

IF (FF (I).GT.0.DO0) $\quad \mathrm{F}=\mathrm{F}+(100 . \mathrm{D} 00+\mathrm{FF}(\mathrm{I})) * \star 2$

ENDDO

RETURN

END

SUBROUTINE HOUGEN ( $\mathrm{A}, \mathrm{M}, \mathrm{F})$

PARAMETER ( $N=13, K=3$ )

IMPLICIT DOUBLE PRECISION (A-H, O-Z)

DIMENSION $X(N, K), \operatorname{RATE}(N), A(*)$

HOUGEN FUNCTION (HOUGEN-WATSON MODEL FOR REACTION KINATICS)

NO. OF PARAMETERS (A) TO ESTIMATE $=5=M$

BEST RESULTS ARE:

$A(1)=1.253031 ; A(2)=1.190943 ; A(3)=0.062798 ; A(4)=0.040063$

$A(5)=0.112453$ ARE BEST ESTIMATES OBTAINED BY ROSENBROCK \&

QUASI-NEWTON METHOD WITH SUM OF SQUARES OF DEVIATION $=0.298900994$

AND $R=0.99945$.

THE NEXT BEST RESULTS GIVEN BY HOOKE-JEEVES \& QUASI-NEWTON

$A(1)=2.475221 ; A(2)=0.599177 ; A(3)=0.124172 ; A(4)=0.083517$

$A(5)=0.217886 ;$ SUM OF SQUARES OF DEVIATION $=0.318593458$

$R=0.99941$

MOST OF THE OTHER METHODS DO NOT PEREORM WELL 


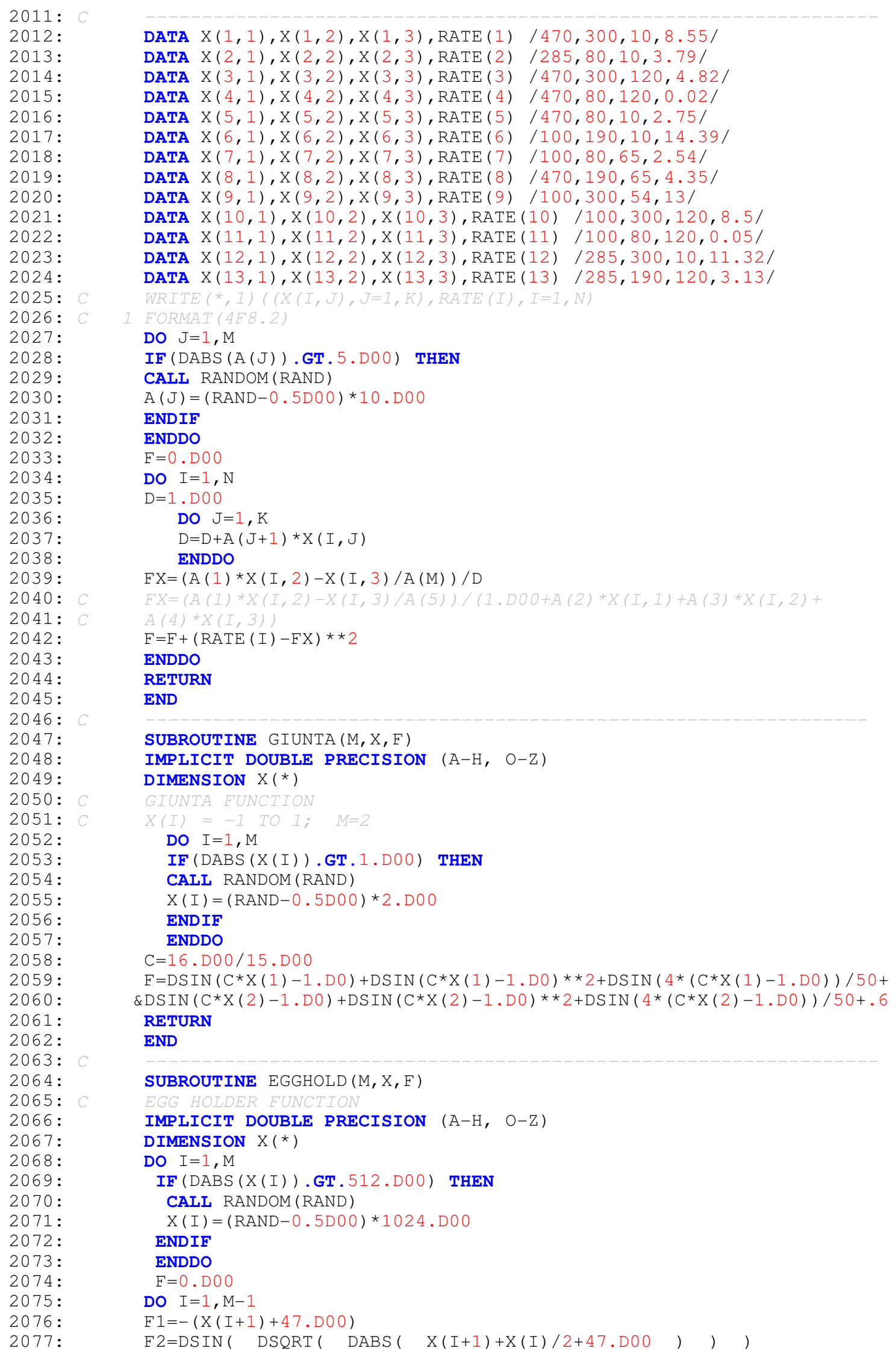




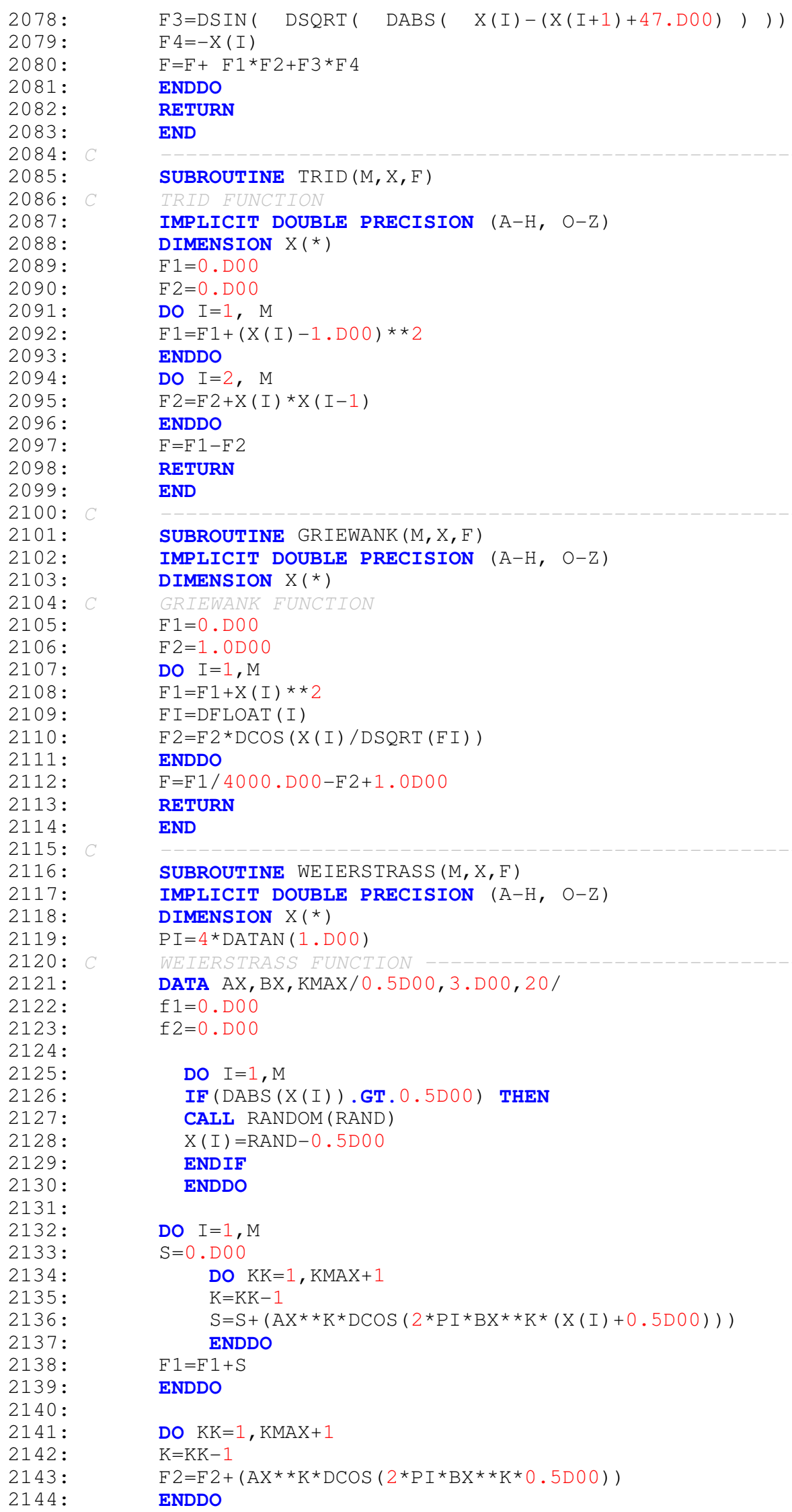




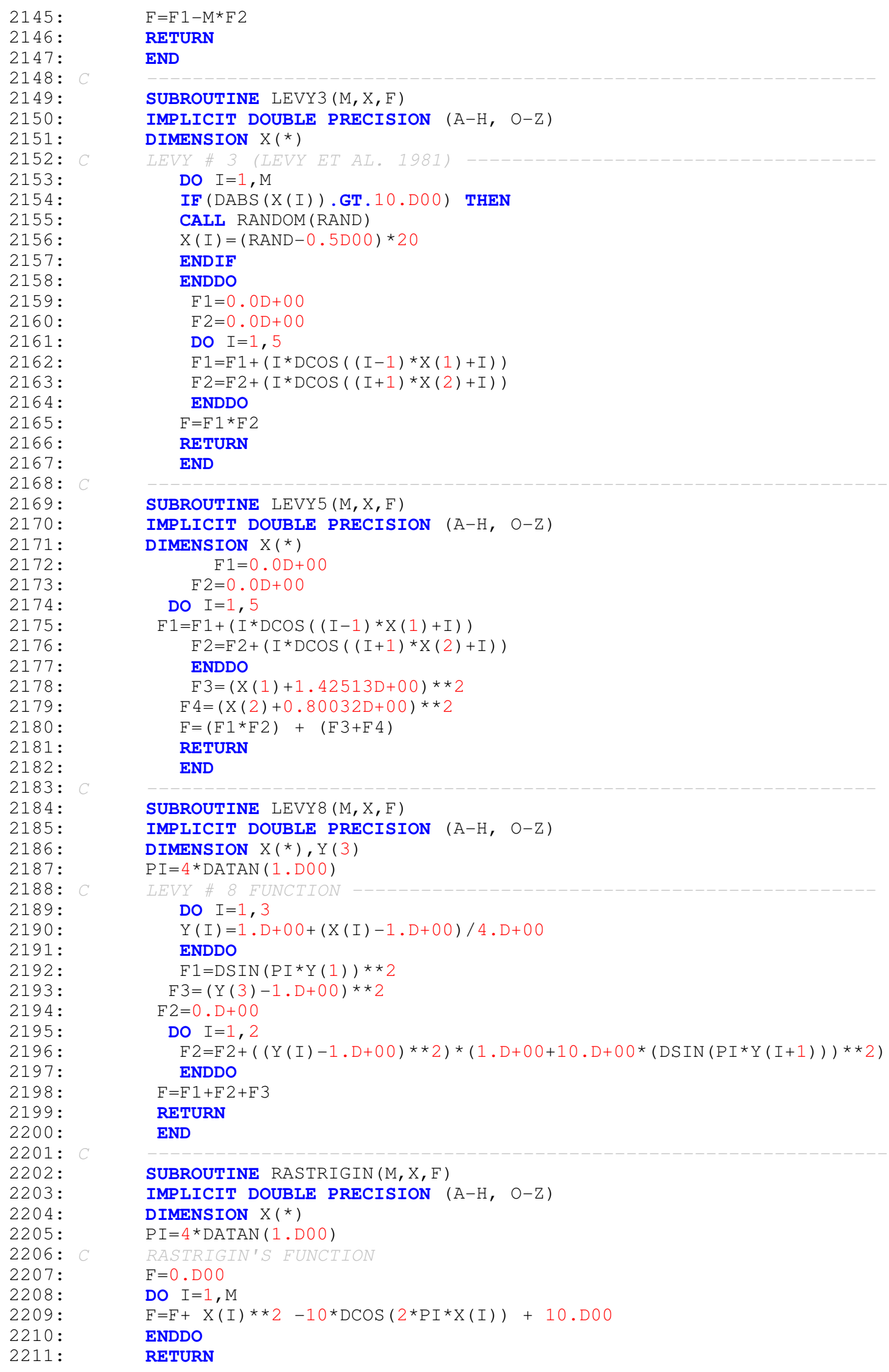




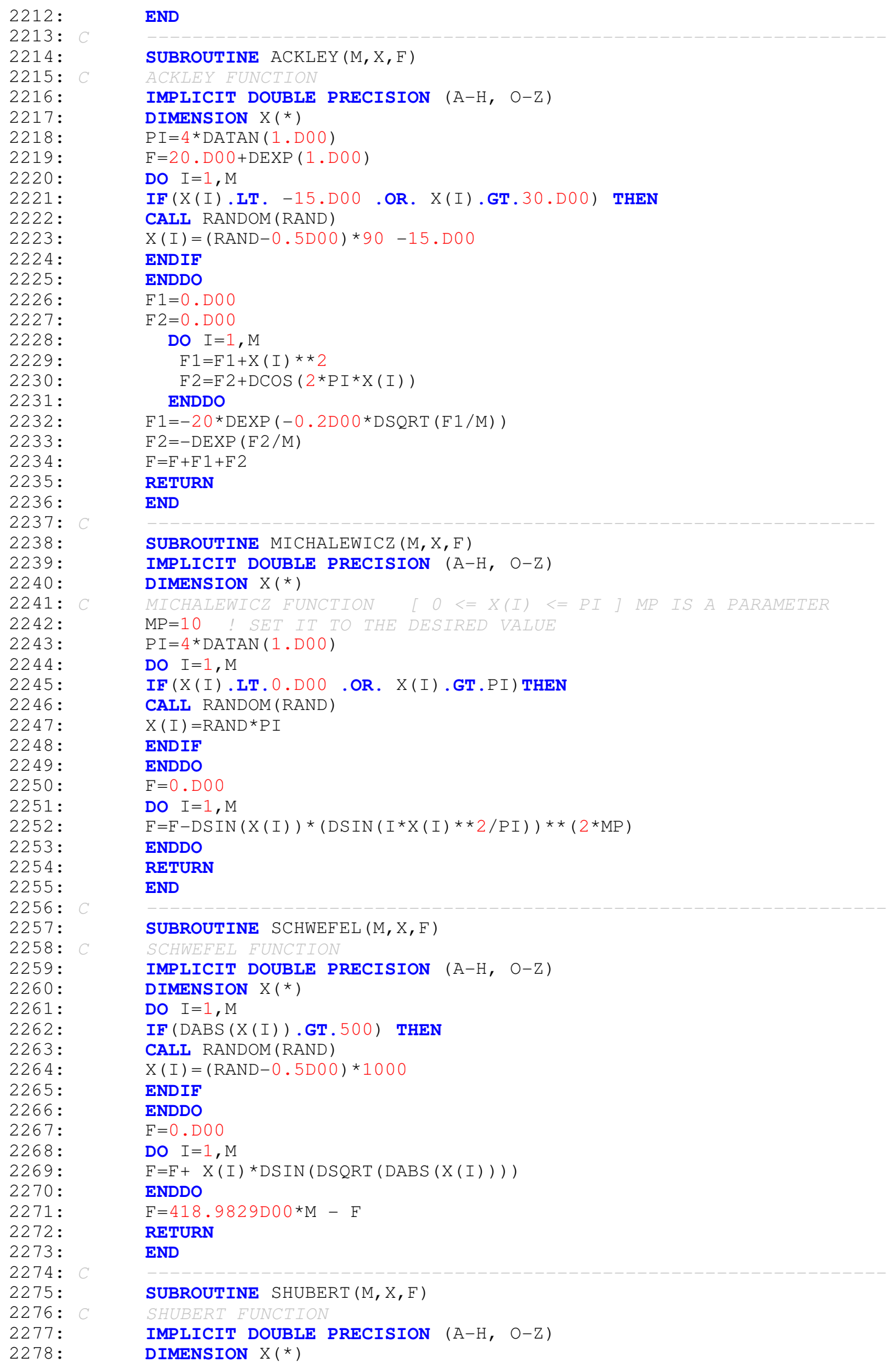




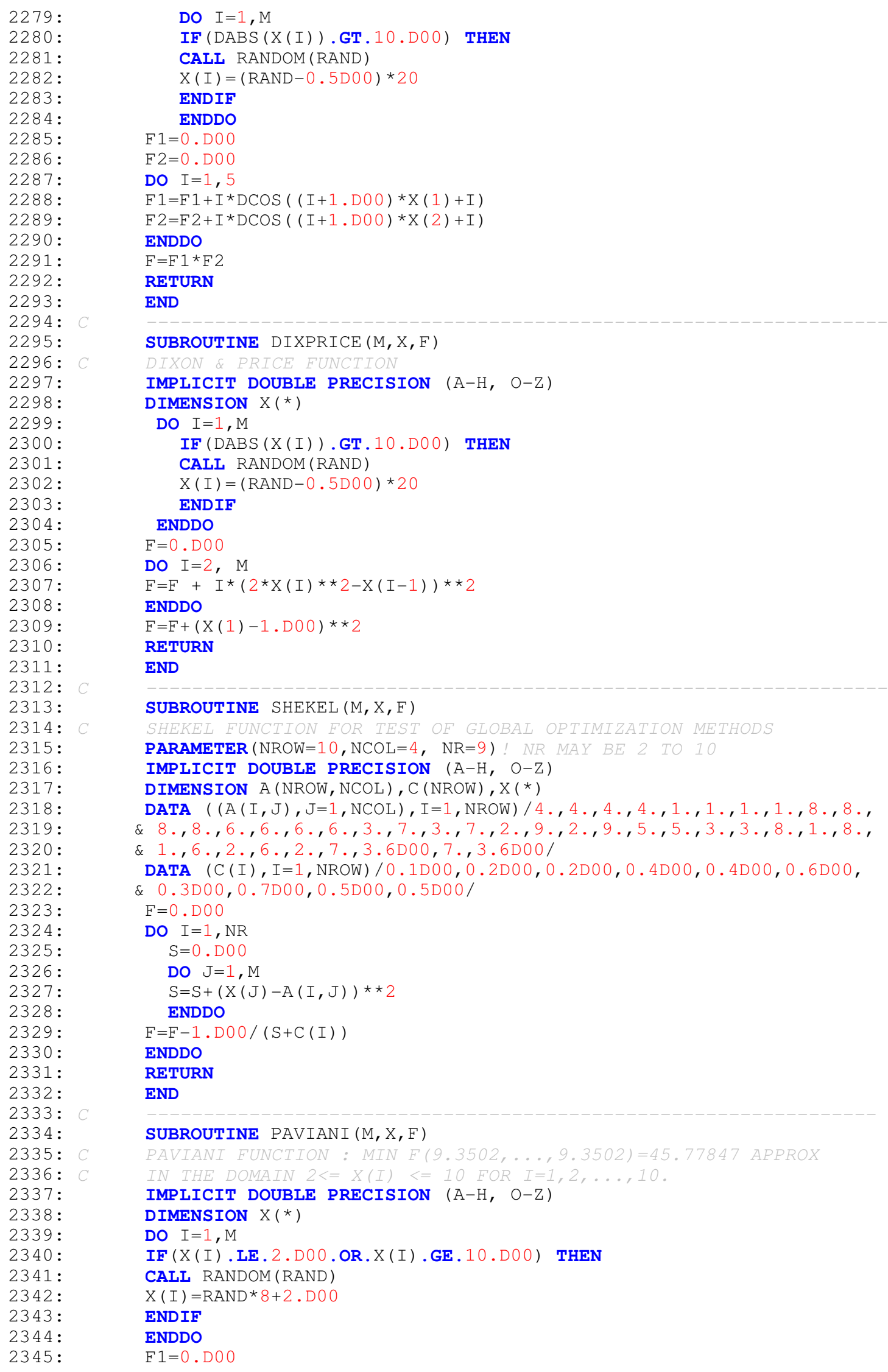




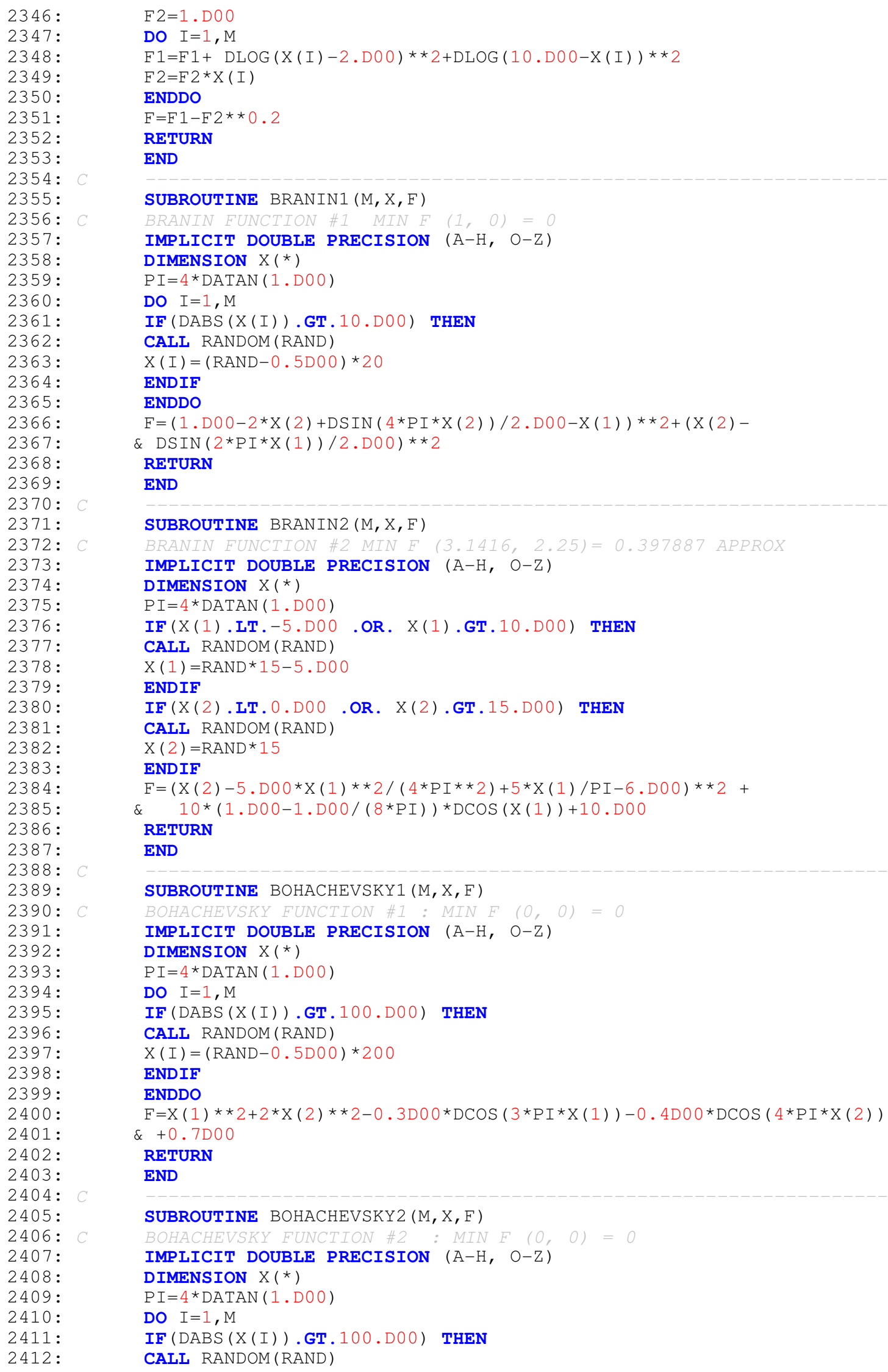




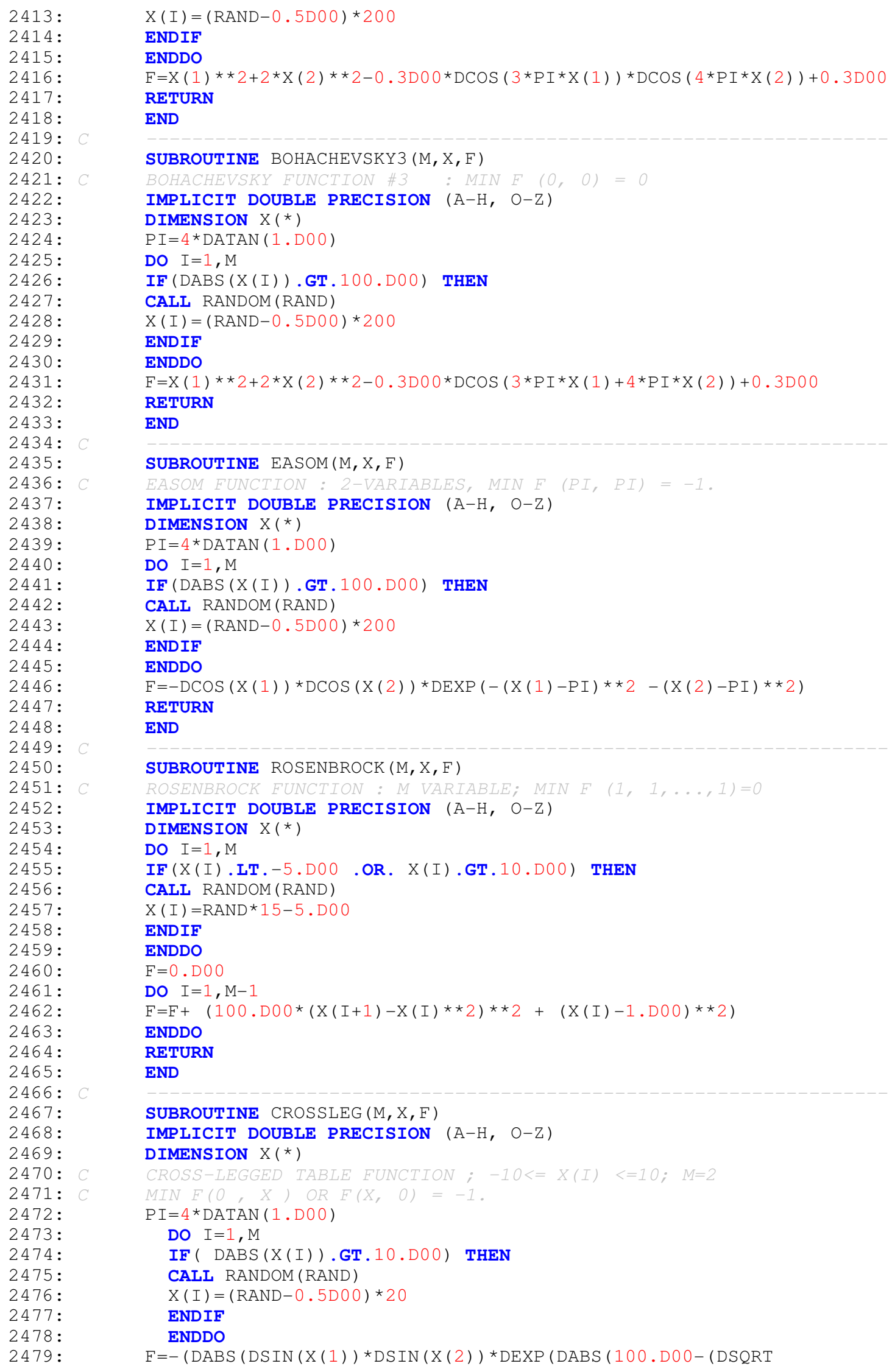




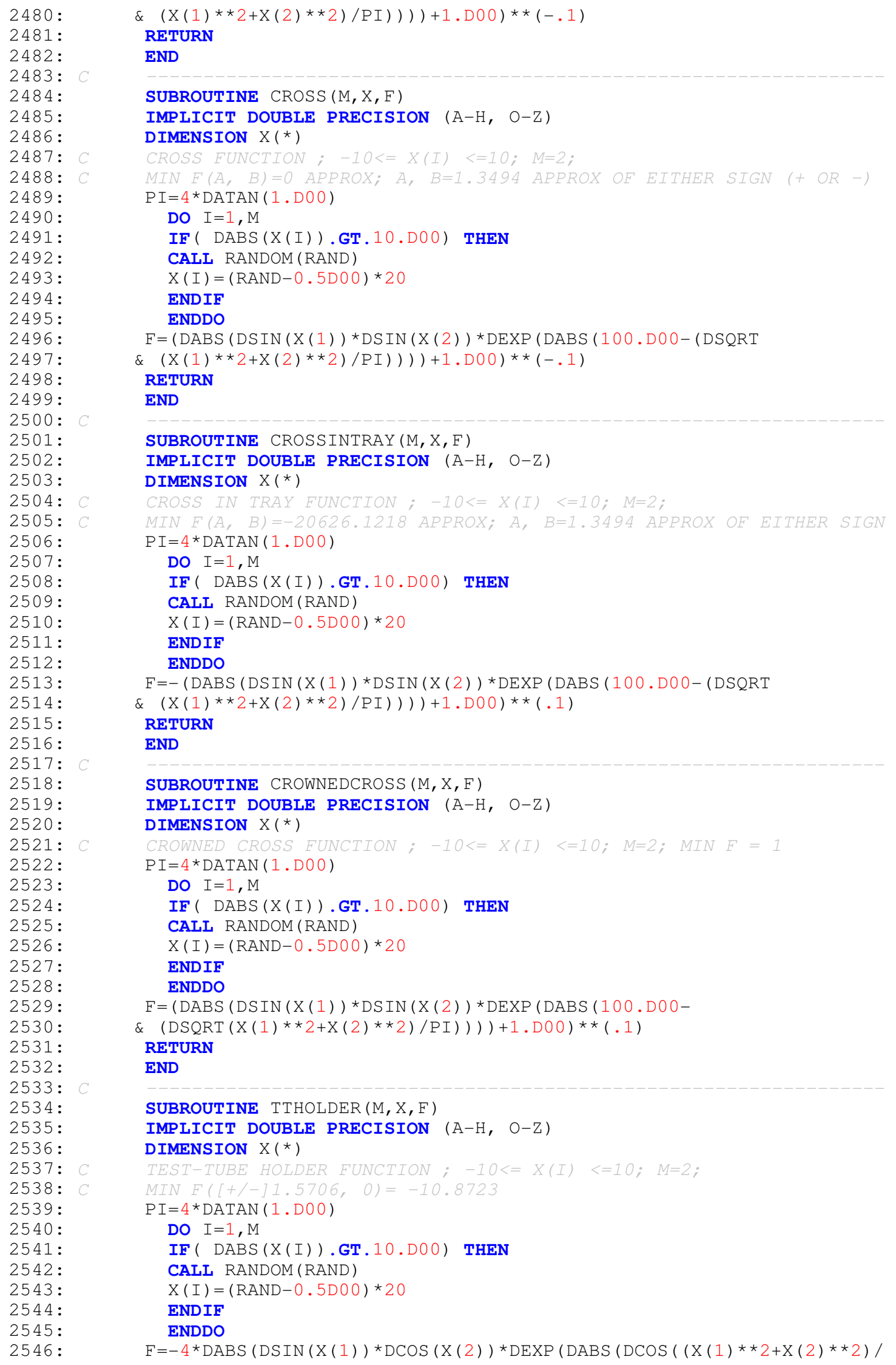




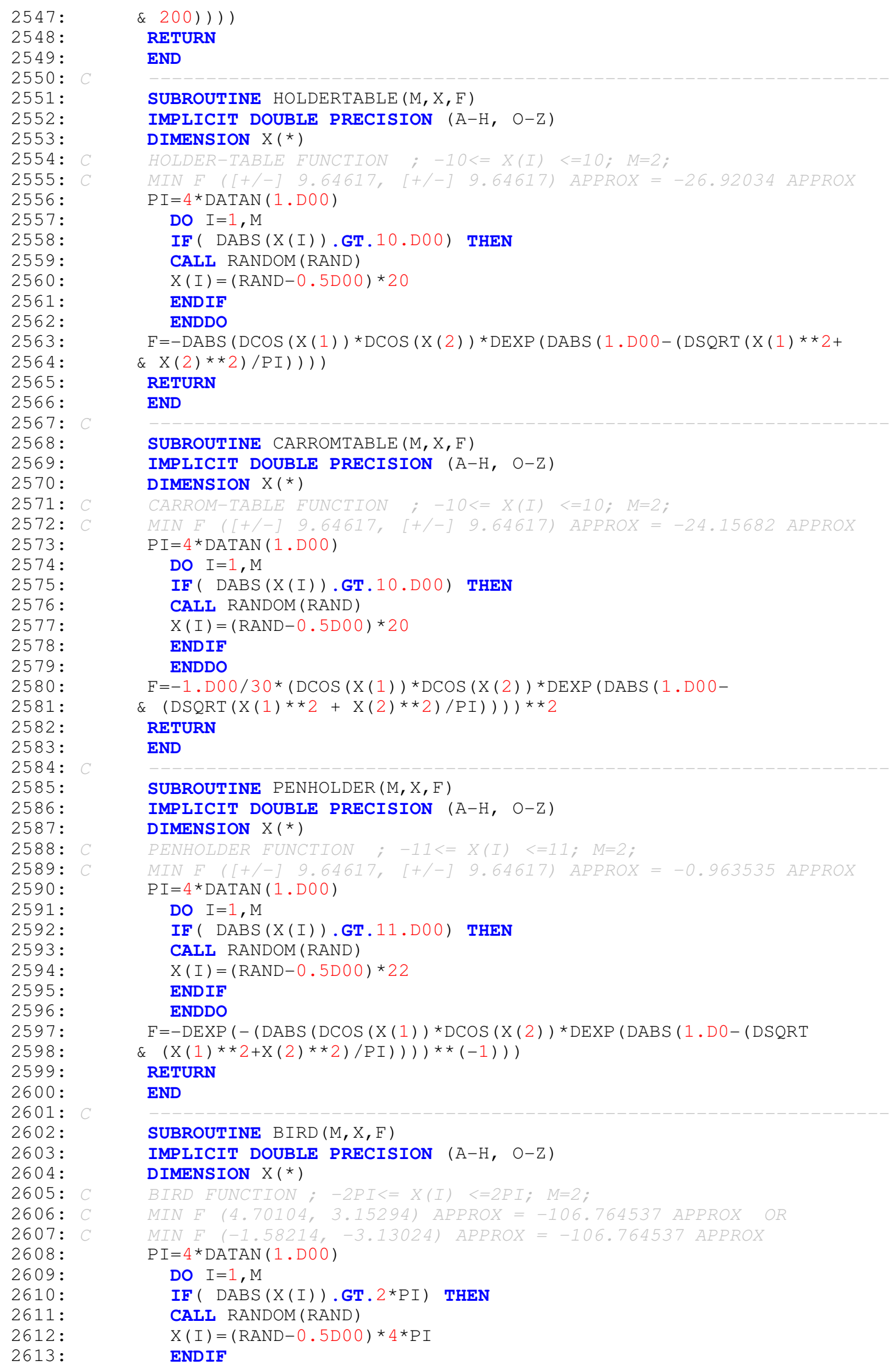




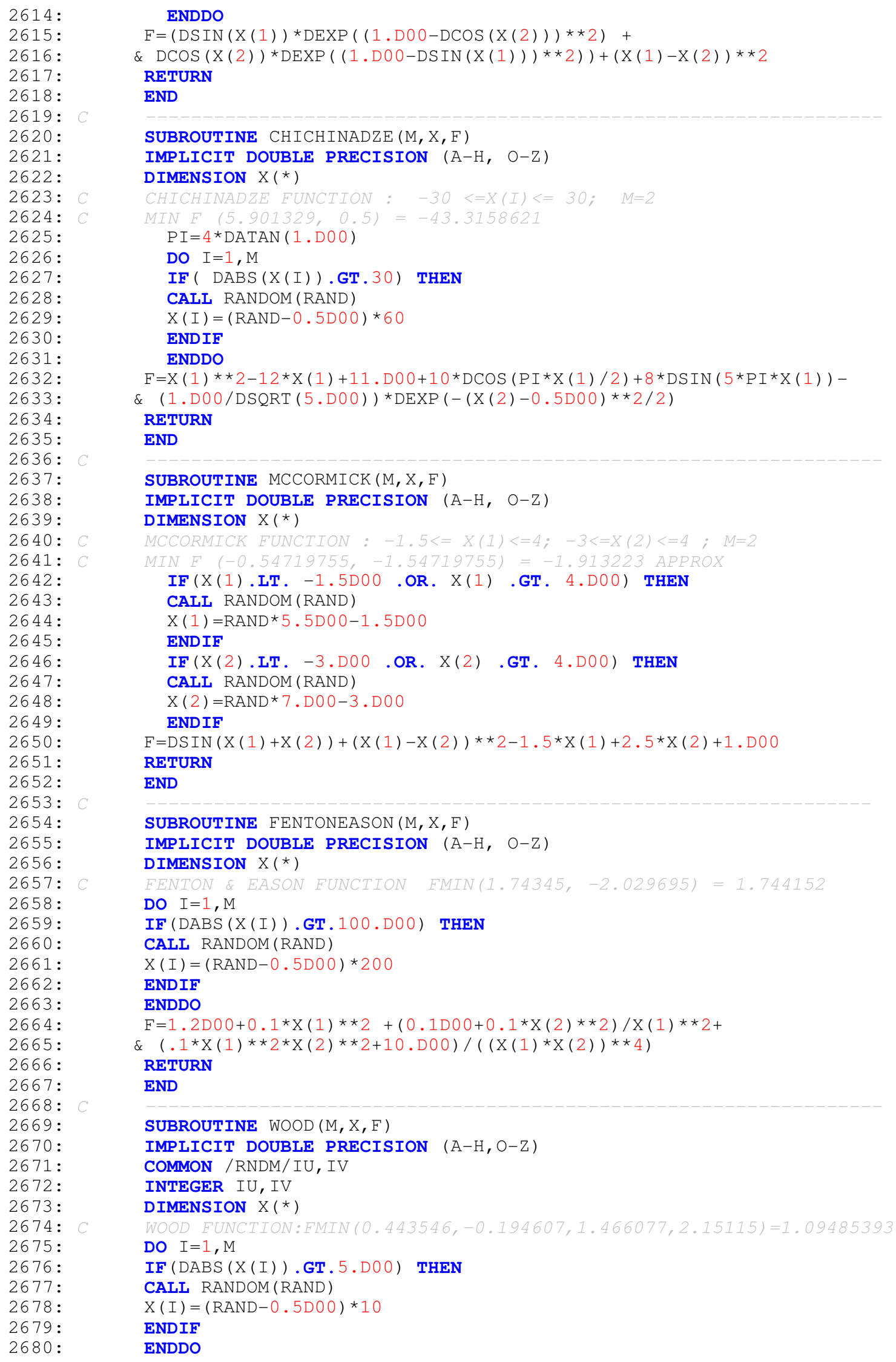




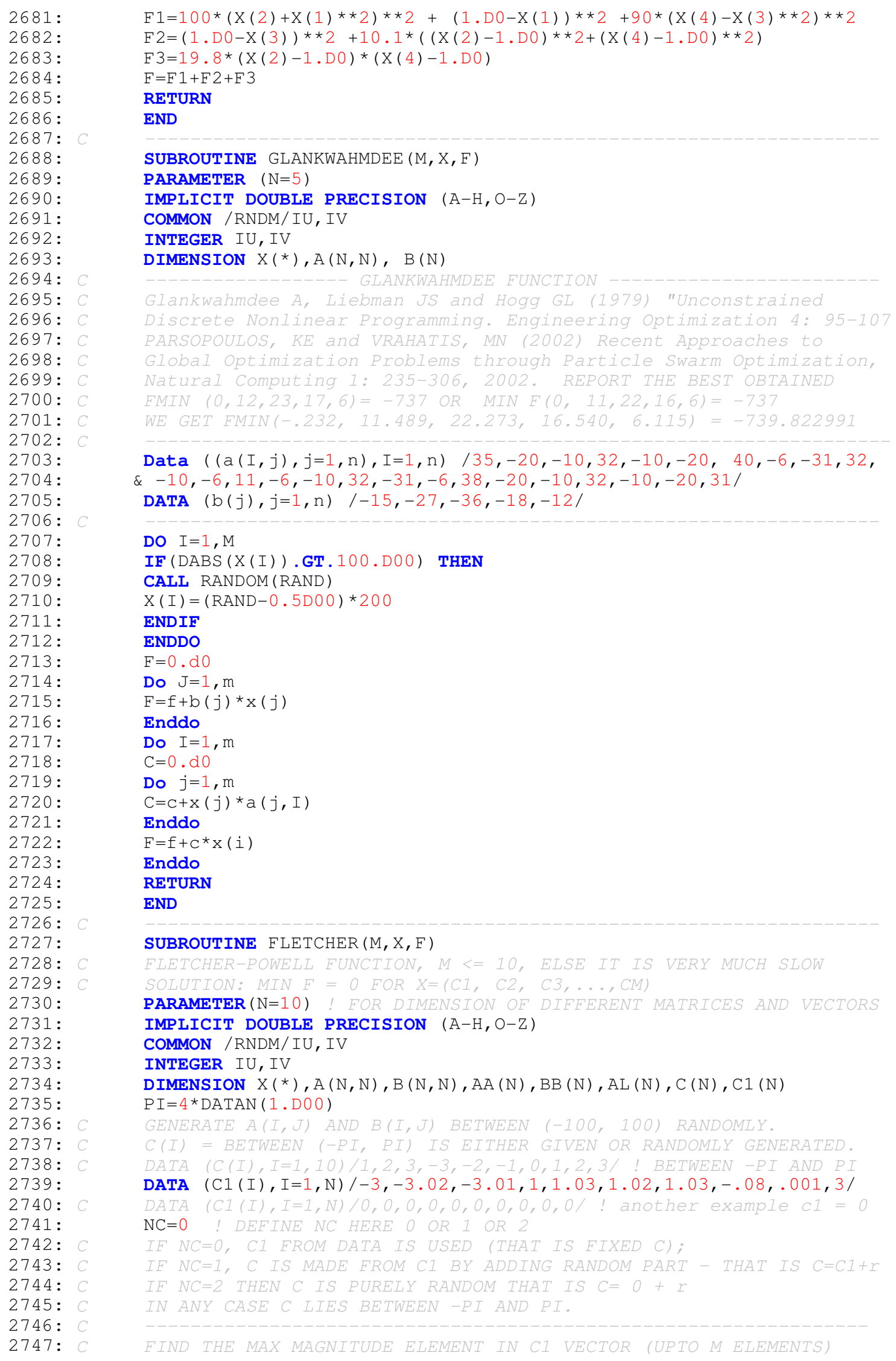




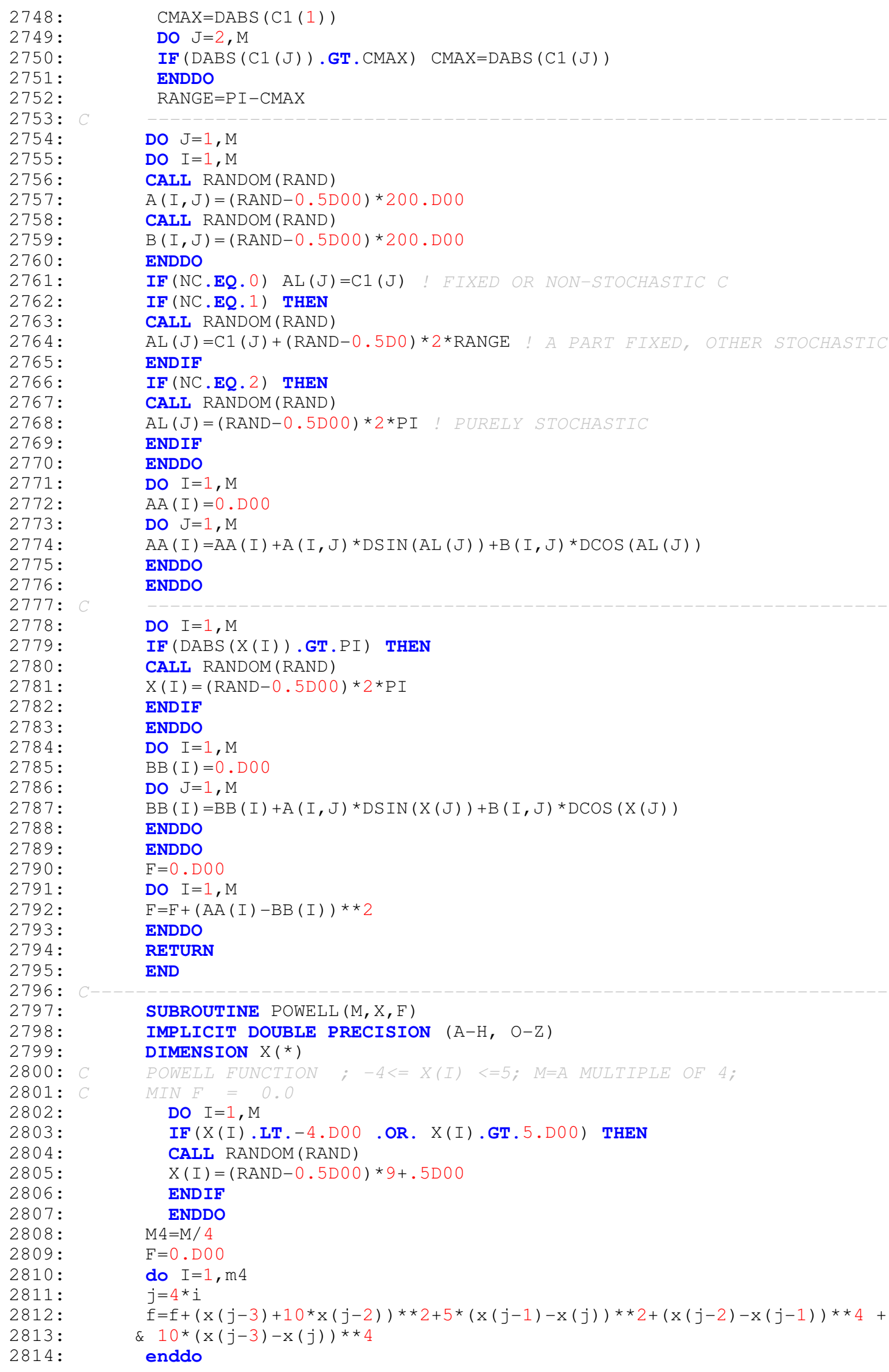




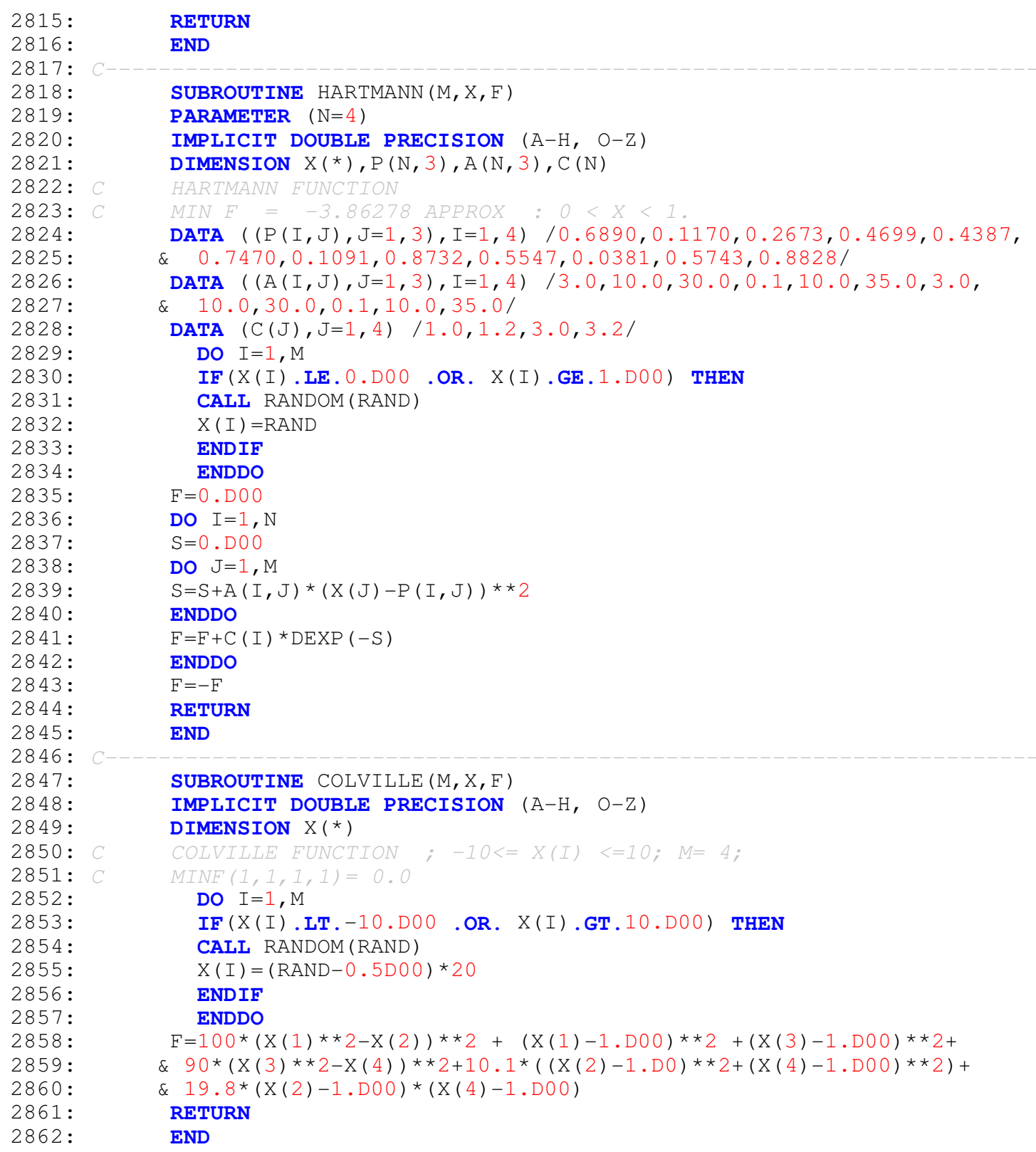

